\title{
Linear ubiquitin assembly complex regulates lung epithelial-driven responses during influenza infection
}

\author{
Patricia L. Brazee, ${ }^{1}$ Luisa Morales-Nebreda, ${ }^{1}$ Natalia D. Magnani, ${ }^{1}$ Joe G. N. Garcia, ${ }^{2}$ Alexander V. Misharin, ${ }^{1}$ Karen M. Ridge, ${ }^{1}$ \\ G.R. Scott Budinger, ${ }^{1}$ Kazuhiro Iwai, ${ }^{3}$ Laura A. Dada, ${ }^{1}$ and Jacob I. Sznajder ${ }^{1}$ \\ 'Division of Pulmonary and Critical Care Medicine, Department of Medicine, Northwestern University, Chicago, Illinois, USA. Department of Medicine, University of Arizona, Tucson, Arizona, USA. \\ ${ }^{3}$ Department of Molecular and Cellular Physiology, Graduate School of Medicine, Kyoto University, Yoshida-konoe-cho, Kyoto, Japan.
}

\begin{abstract}
Influenza A virus (IAV) is among the most common causes of pneumonia-related death worldwide. Pulmonary epithelial cells are the primary target for viral infection and replication and respond by releasing inflammatory mediators that recruit immune cells to mount the host response. Severe lung injury and death during IAV infection result from an exuberant host inflammatory response. The linear ubiquitin assembly complex (LUBAC), composed of SHARPIN, HOIL-1L, and HOIP, is a critical regulator of NF-кB-dependent inflammation. Using mice with lung epithelial-specific deletions of HOIL-1L or HOIP in a model of IAV infection, we provided evidence that, while a reduction in the inflammatory response was beneficial, ablation of the LUBAC-dependent lung epithelial-driven response worsened lung injury and increased mortality. Moreover, we described a mechanism for the upregulation of HOIL-1L in infected and noninfected cells triggered by the activation of type I IFN receptor and mediated by IRF1, which was maladaptive and contributed to hyperinflammation. Thus, we propose that lung epithelial LUBAC acts as a molecular rheostat that could be selectively targeted to modulate the immune response in patients with severe IAV-induced pneumonia.
\end{abstract}

\section{Introduction}

Seasonal influenza A viral infection affects a significant proportion of the population in the United States and worldwide. While most patients infected with influenza A virus (IAV) recover without major sequelae, severe viral pneumonia is one of the most common causes of acute respiratory distress syndrome (ARDS) (1-4). Impairment of gas exchange in IAV-induced ARDS, in large part, is due to damage to the alveolar epithelial barrier and edema accumulation (1, 4-6). During IAV infection an exaggerated inflammatory response, known as "cytokine storm," can occur, which disrupts the alveolar epithelial barrier and leads to the development of ARDS (5), increasing IAV-induced morbidity and mortality.

Alveolar epithelial cells (AECs) are targets of IAV infection and orchestrate the host immune response necessary for viral clearance $(7,8)$. As IAV replicates within AECs, viral particles accumulate in the cytosol and are sensed by the host pattern recognition receptor retinoic acid-inducible gene I (RIG-I), inducing the production of cytokines and IFNs (9-11). AEC-derived cytokines and IFNs promote recruitment of host immune cells, which release additional cytokines and proteases in an effort to limit viral spread (5). However, the nonspecific nature of the innate immune response can damage noninfected adjacent cells $(5,7)$. As AECs die, either by direct infection or as a consequence of the inflammatory response, the alveolar barrier becomes permeable to solutes, leading to edema accumulation and impaired

Conflict of interest: The authors have declared that no conflict of interest exists. Copyright: () 2020, American Society for Clinical Investigation.

Submitted: March 6, 2019; Accepted: November 6, 2019; Published: January 27, 2020.

Reference information: / Clin Invest. 2020;130(3):1301-1314.

https://doi.org/10.1172/JCl128368. gas exchange $(5,7,12)$. Data from animal models of severe IAV infection suggest that cytokine storm is the major driver of morbidity and mortality (13-17). Several studies suggest that reductions in circulating cytokines improve lung injury and survival, highlighting the importance of modulating the host inflammatory response during IAV infection $(14,18,19)$.

The linear ubiquitin assembly complex (LUBAC) is essential for $\mathrm{NF}-\kappa \mathrm{B}$ activation, targeting the $\mathrm{NF}-\kappa \mathrm{B}$ essential modulator (NEMO) for linear ubiquitination (20-24). LUBAC is an E3 ligase complex composed of the accessory protein Shank-associated RH domain-interacting protein (SHARPIN) as well as two RINGin-between-RING ligases: heme-oxidized iron-responsive element-binding protein 2 ubiquitin ligase-1L (HOIL-1L) and the catalytically active component HOIL-1-interacting protein (HOIP) $(20,25,26)$. Unlike most E3 ubiquitin ligases, which utilize the internal lysine residues of ubiquitin, LUBAC forms Met-1-linked head-to-tail linear ubiquitin chains $(20,22,25)$. While the exact stoichiometry of the $600-\mathrm{kDa}$ complex has yet to be elucidated, the presence of all 3 LUBAC components is necessary for its stability and maximal activity, despite HOIP being the only catalytically active component of the complex $(25,27)$. Predominantly studied downstream of the TNF receptor superfamily (TNFRSF), linear ubiquitin chains on NEMO stabilize the IKB kinase (IKK) complex, consisting of NEMO, IKK $\alpha$, and IKK $\beta$, enabling the phosphorylation and degradation of $\mathrm{I} \kappa \mathrm{B} \alpha$, releasing $\mathrm{NF}-\kappa \mathrm{B}$ to translocate to the nucleus, and stimulating downstream inflammation $(21,28$, 29). NEMO can also form a complex that activates IFN regulatory factor 3 (IRF3), initiating its dimerization and translocation to the nucleus, where it drives type I IFN production to modulate the antiviral response (30). While the role of LUBAC in NF- $\mathrm{KB}$ signaling downstream of TNFRSF has been well established, the role of 
A

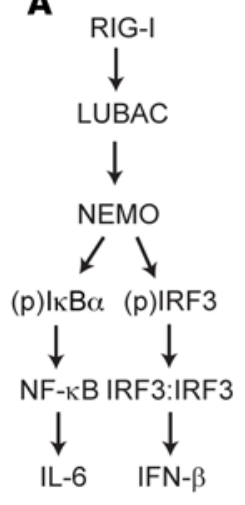

B

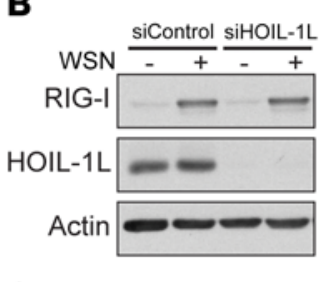

C

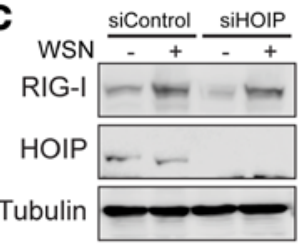

D

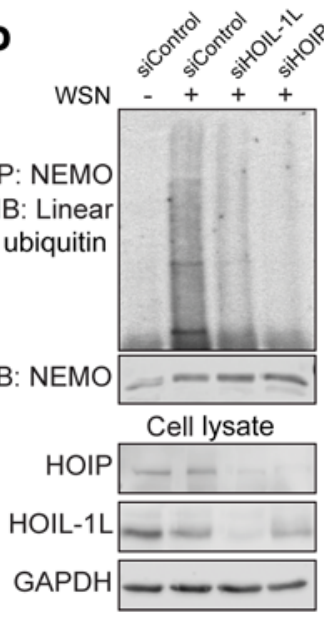

Figure 1. Linear ubiquitin chains modulate the NF- $\mathrm{B}$ response to IAV downstream of RIG-I signaling in vitro. (A) Simplified signaling pathway. (B-C) A549 cells were infected with WSN (1 MOI, 16 hours) after transfection with nontargeting siRNA (siControl) or siRNA against either HOIL-1L or HOIP. (B-E) Representative immunoblots of RIG-I activation $(n=4)(B-C)$, linear ubiquitination of NEMO $(n=3)(\mathbf{D})$, and p-IkB $\alpha$, total IkB $\alpha$, plRF3, and total IRF3 $(n=4)(E)$. Means \pm SD overlaid with individual data points representing replicates are depicted; ${ }^{*} P<0.05,{ }^{* * *} P<0.0001$ (1-way ANOVA, Bonferroni post hoc test).
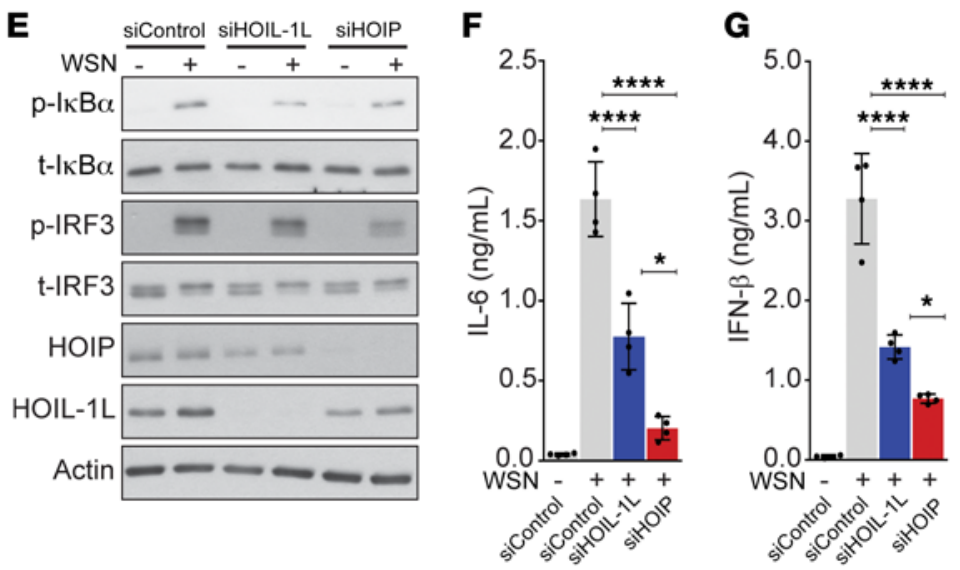

alveolar epithelial LUBAC in regulating NF- $\mathrm{kB}$-dependent inflammation downstream of RIG-I during IAV infection is unknown.

In this study, we report that the degree of LUBAC activity determines the alveolar epithelial-driven inflammatory response to IAV infection. During IAV infection, type I IFN upregulates HOIL-1L expression in AECs and thus enhances LUBAC formation, leading to an exaggerated inflammatory response and severe lung injury. In agreement with a role of alveolar epithelial LUBAC driving the lung inflammatory response, reducing formation of the complex by using transgenic mice with a lung epithelial cellspecific deletion of HOIL-1L protected the mice from severe lung injury and increased survival. Moreover, complete loss of lung epithelial LUBAC activity, as observed in mice with a genetic deletion of HOIP from the lung epithelium, worsened outcomes during IAV infection. This work uncovers a novel role for LUBAC as a rheostat of the epithelial-driven inflammatory response to IAV infection, which could be targeted as a potential therapeutic strategy for control of IAV-induced cytokine storm to improve patient outcomes during severe infection.

\section{Results}

Linear ubiquitin chains regulate the $N F-\kappa B$ response to IAV downstream of RIG-I signaling. We infected an alveolar epithelial cell line (A549) with H1N1 A/WSN/1933 (WSN) at 1 MOI, for 16 hours, a dose and time at which we observed RIG-I-dependent $\mathrm{NF}-\kappa \mathrm{B}$ and IRF3 activation (measured by the phosphorylation relative to total I $\mathrm{B} \alpha$ and IRF3, respectively), NEMO ubiquitination, and cytokine production (Figure 1 and Supplemental Figure 1, A-C; supplemental material available online with this article; https://doi.org/10.1172/JCI128368DS1). After siRNA silencing of either HOIL-1L or HOIP, we detected no appreciable differences in RIG-I activation in comparison with control siRNA-treated cells in response to WSN infection (Figure 1, B and C, and Supplemental Figure 1, D and E). However, analysis of NEMO ubiquitination after immunoprecipitation from cell lysates showed that loss of LUBAC components resulted in a graded decrease of linear chains, where silenced HOIL-1L reduced their abundance and siHOIP inhibited the linear ubiquitin of NEMO during IAV infection (Figure 1D). Downstream of NEMO linear ubiquitination, silencing of HOIL-1L or HOIP decreased the IAV-induced activation of NF- $\mathrm{BB}$ and IRF3 as well as the secretion of IL- 6 and IFN- $\beta$ in a similarly graded manner (Figure 1, E-G, and Supplemental Figure 1, F and $G$ ). Together these results suggest that LUBAC regulates the inflammatory and antiviral response downstream of RIG-I signaling in response to IAV.

Loss of HOIL-1L from the lung epithelium reduces lung injury and improves survival in mice infected with IAV. To evaluate whether LUBAC activity in the lung epithelium regulates IAV-induced lung injury, we generated mice with a tissue-specific deletion of full-length HOIL-1L (SPC ${ }^{\text {Cre }} /$ HOIL-1L ${ }^{\mathrm{fl} / \mathrm{fl}}$ ) (31). These mice were generated using the same construct described for the well-defined HOIL-1L $\mathrm{L}^{-/-}$mice $(21,31)$, which do not express full-length HOIL-1L 
A

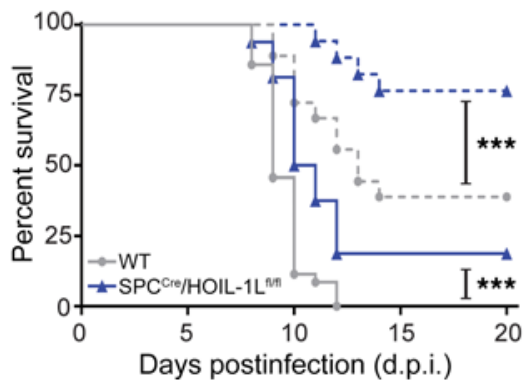

B

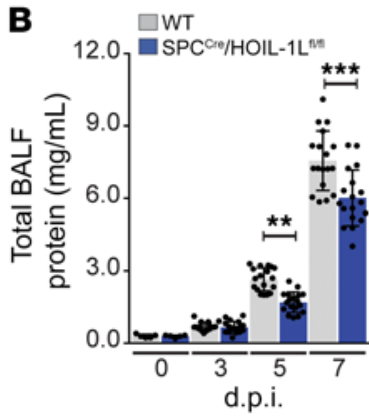

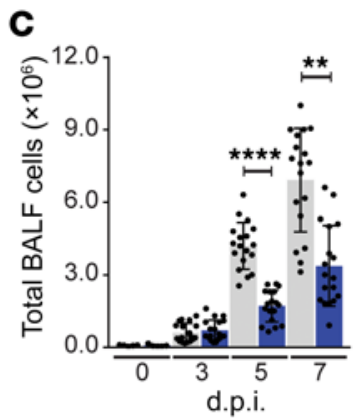

D

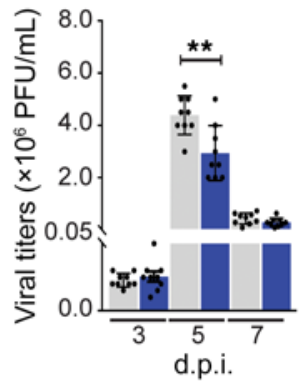

Figure 2. Loss of HOIL-1L from the alveolar epithelium improves survival and reduces lung injury in mice infected with IAV. (A) Survival of WT and SPC Cre $/$ HOIL-1L $^{\mathrm{fl} / \mathrm{fl}}$ mice infected with a low $(n=18$, dashed line) or lethal $(n=30$, solid line) dose of WSN. (B and C) BALF was collected at 0 ( $n=5)$, 3, 5, and 7 d.p.i. $(n=18)$ with a lethal dose of WSN and analyzed for total protein concentration (B) and cellular infiltration (C). (D) Quantification of viral titers in WT and SPCCre $/ \mathrm{HOIL}-1 \mathrm{~L}^{\mathrm{fl} / \mathrm{fl}}$ mice at 3,5 , and 7 d.p.i. $(n=9)$. Means $\pm \mathrm{SD}$ overlaid with individual data points representing replicates are depicted; ${ }^{* *} P<0.01$, ${ }^{* * *} P<0.005,{ }^{* * * *} P<0.0001$ (A, 2-tailed log-rank Mantel-Cox test; B-D, 1-way ANOVA, Bonferroni post hoc test).

but instead express a $30-\mathrm{kDa}$ truncated variant (21) that results in reduced levels of 600-kDa LUBAC (32) as well as impaired NF-kB signaling (21). Immunoblots of mouse alveolar type 2 (AT2) cell lysates using a specific antibody against either the C-terminus (21, 31) or the N-terminus of HOIL-1L (33) showed a band at approximately $55 \mathrm{kDa}$ in WT AT2 cells, corresponding to the molecular weight of full-length HOIL-1L, that was absent in SPC ${ }^{\text {Cre }} / \mathrm{HOIL}-$ $1 \mathrm{~L}^{\mathrm{f} / \mathrm{l}}$ AT2 cell lysates (Supplemental Figure 2, A and B). Additionally, using the HOIL-1L N-terminus antibody, we observed a low-abundance band at approximately $30 \mathrm{kDa}$ in SPC $\mathrm{Sre}^{\mathrm{Cr}} / \mathrm{HOIL}-$ $1 \mathrm{~L}^{\mathrm{f} / \mathrm{l}}$ AT2 cells (Supplemental Figure 2A and ref. 33). This observation is in agreement with the description of the $30-\mathrm{kDa}$ band in lysates from HOIL-1 $\mathrm{L}^{-1-}$ mice, which represents a truncated splice variant of HOIL-1L (N-HOIL-1L) lacking the C-terminal RING domain but retaining its ubiquitin-like (UBL) domain for interaction with HOIP as well as its LUBAC-tethering motif (LTM) necessary for interactions with SHARPIN $(21,33,34)$. The absence of HOIL-1L can destabilize the complex and decrease HOIP and SHARPIN expression $(27,33,35)$; however, we observed no significant change in HOIP expression between WT and $\mathrm{SPC}^{\mathrm{Cre}} /$ HOIL-1L ${ }^{\mathrm{f} / \mathrm{ll}}$ AT2 cells (Supplemental Figure 2B). Interestingly, we observed a slight increase in SHARPIN expression in SPC Cre / HOIL-1L ${ }^{\mathrm{A} / \mathrm{f}}$ AT2 cells compared with WT (Supplemental Figure 2B). SHARPIN is sufficient to stabilize HOIP and promote LUBAC activity in the absence of HOIL-1L, albeit with lower efficiency compared with complexes containing HOIL-1L (27), supporting the notion that respiratory epithelial cells in SPC Cre $/ H O I L-1 L^{\mathrm{H} / \mathrm{I}}$ mice retain some degree of LUBAC activity.

WT and SPC ${ }^{\text {Cre }} / \mathrm{HOIL}-1 \mathrm{~L}^{\mathrm{A} / \mathrm{f}}$ mice were infected intratracheally with a low or lethal dose of WSN and monitored for survival. Low-dose WSN infection resulted in approximately $75 \%$ survival of SPC Cre $/$ HOIL-1L $1 \mathrm{~L}^{\mathrm{A} / \mathrm{l}}$ mice compared with $40 \%$ of WT mice at 20 days after infection (d.p.i.) (Figure 2A). A lethal dose of WSN resulted in $100 \%$ mortality of WT mice by 12 d.p.i., while approximately $20 \%$ of SPC Cre $/$ HOIL- $1 \mathrm{~L}^{\mathrm{n} / \mathrm{l}}$ mice survived. The differences in mortality during infection with WSN corresponded with decreased severity of lung injury in SPC Cre $/ \mathrm{HOIL}-1 \mathrm{~L}^{\mathrm{t} / \mathrm{l}}$ mice, assessed by measurement of total protein content in bronchoalveolar lavage fluid (BALF), which was decreased by approximately $25 \%$ at 5 d.p.i. and $20 \%$ at 7 d.p.i. with the lethal dose in comparison with WT mice (Figure 2B). In agreement with reduced barrier permeability, at the same time points we detected a significant decrease in total cell count in BALF in SPC ${ }^{\mathrm{Cre}} / \mathrm{HOIL}-1 \mathrm{~L}^{\mathrm{I} / \mathrm{ll}}$ mice as compared with WT mice (Figure 2C). Careful examination of multiple parameters in H\&E-stained lung sections, including peribronchial infiltration, bronchial exudate, alveolar infiltration, and thickening of alveolar walls, showed reduced severity of lung injury in SPC $\mathrm{Cre}^{\mathrm{H}} / \mathrm{HOIL}-1 \mathrm{~L}^{\mathrm{t} / \mathrm{Al}}$ mice as compared with WT at 7 d.p.i. with low-dose WSN (Supplemental Figure 2, C and D). Moreover, in WT mice we observed that a lethal dose of WSN resulted in peak viremia at 5 d.p.i., which was decreased by $27 \%$ in SPC Cre $/ H O I L-1 L^{\mathrm{f} / \mathrm{l}}$ mice with no significant differences in clearance at 7 d.p.i. (Figure 2D).

BALF samples from WT and SPC ${ }^{\text {Cre }} / \mathrm{HOIL}-1 \mathrm{~L}^{\mathrm{f} / \mathrm{l} l}$ mice were further analyzed to determine cytokine content after lethal WSN infection. WT mice infected with WSN had high levels of IL-6 (Figure 3A), MCP-1, and IFN- $\gamma$ by 5 d.p.i. that remained elevated through 7 d.p.i., all of which were significantly reduced in the BALF of WSN-infected SPC ${ }^{\mathrm{Cre}} / \mathrm{HOIL}-1 \mathrm{~L}^{\mathrm{A} / \mathrm{ll}}$ mice (Supplemental Figure 2, $\mathrm{E}$ and $\mathrm{F}$ ), while BALF levels of TNF- $\alpha$ were similarly elevated in both strains (Supplemental Figure 2G). Additionally, at 3 and 5 d.p.i., type I IFNs (IFN- $\alpha$, IFN- $\beta$ ) were significantly reduced in SPC ${ }^{\text {Cre }}$ / HOIL-1L $\mathrm{L}^{\mathrm{A} / \mathrm{f}}$ compared with WT mice (Figure 3, B and C). Conversely, at 7 d.p.i., BALF from SPC Cre/HOIL-1L $\mathrm{L}^{\mathrm{t} / \mathrm{l}}$ mice contained 2-fold higher levels of the antiinflammatory cytokine IL-10 (Supplemental Figure 2H). At 5 d.p.i. we also observed reduced levels of IL-1 $\beta$ in the BALF of SPC Cre $/$ HOIL-1L $1 / \mathrm{A} / \mathrm{ll}$ mice compared with WT mice (Supplemental Figure 2I) that corresponded with reduced Illb transcription in isolated SPC ${ }^{\text {Cre }} / \mathrm{HOIL}-1 \mathrm{~L}^{\mathrm{f} / \mathrm{l}}$ AT2 cells (Supplemental Figure 2J) with no defect in SPC ${ }^{\text {Cre }} / \mathrm{HOIL}-1 \mathrm{~L}^{\mathrm{f} / \mathrm{l} / \mathrm{l}}$ AT2 inflammasome activation compared with WT AT2 cells at 5 d.p.i. (Supplemental Figure $2 \mathrm{~K}$ ). Taken together, these results suggest that loss of fulllength HOIL-1L from lung epithelial cells confers protection during IAV infection by altering the ratio of proinflammatory and antiinflammatory cytokines and reducing the levels of IFNs.

AEC-derived cytokines and IFNs promote recruitment and activation of immune cells (5). To determine the effect of loss of HOIL-1L on the innate immune response, we analyzed lung myeloid and lymphoid populations in $\mathrm{WT}$ and $\mathrm{SPC}^{\mathrm{Cre}} / \mathrm{HOIL}-1 \mathrm{~L}^{\mathrm{A} / \mathrm{fl}}$ lungs at $0,3,5$, and 7 d.p.i. by flow cytometry using defined lineage-specific cell surface markers and gated as described in Methods and 

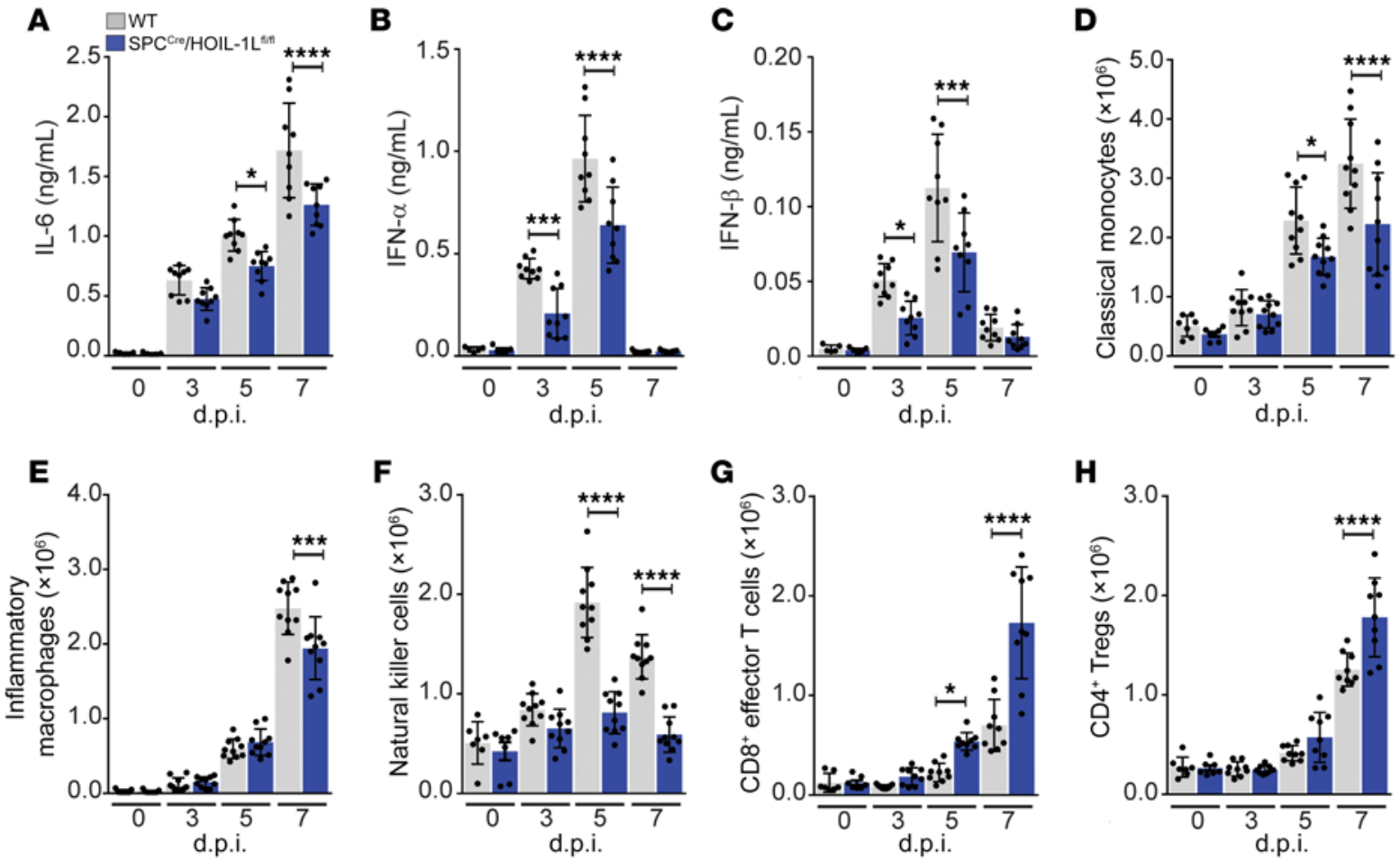

Figure 3. Alveolar epithelial loss of HOIL-1L reduces the inflammatory response in IAV-infected mice. WT and SPCCre $/ \mathrm{HOIL}^{\mathrm{C}}-\mathrm{L}^{\mathrm{fl} / \mathrm{fl}}$ mice were infected with a lethal dose of WSN. (A-C) BALF at $0(n=5), 3,5$, and 7 d.p.i. $(n=9)$ was analyzed by ELISA for IL-6 (A), IFN- $\alpha(\mathbf{B})$, and IFN- $\beta(\mathbf{C})$. (D-H) Lung immune cell

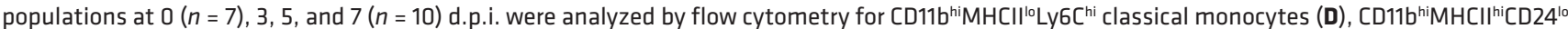

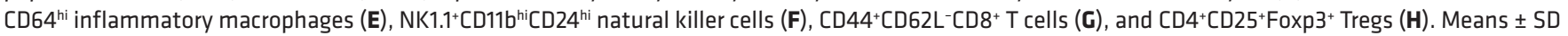
overlaid with individual data points representing replicates are depicted; ${ }^{*} P<0.05,{ }^{* *} P<0.005,{ }^{* * *} P<0.0001$ (1-way ANOVA, Bonferroni post hoc test).

Supplemental Figure 3, A and B (36). In WT mice, we observed a significant increase in the number of $\mathrm{CD} 11 \mathrm{~b}^{\text {hi }} \mathrm{MHCII}^{\mathrm{lo}} \mathrm{Ly} 6 \mathrm{C}^{\text {hi }}$ classical monocytes after WSN infection, which was blunted in $\mathrm{SPC}^{\mathrm{Cre}}$ / HOIL-1L $\mathrm{L}^{\mathrm{fl} / \mathrm{fl}}$ mice at all time points (Figure 3D). Similarly, recruitment of CD11b ${ }^{\text {hi }} \mathrm{MHCII}{ }^{\text {hi }} \mathrm{CD} 24^{\text {lo }} \mathrm{CD} 64^{\text {hi }}$ monocyte-derived inflammatory macrophages was decreased by $45 \%$ in SPC $\mathrm{Cre}^{\mathrm{CHOIL}}-1 \mathrm{~L}^{\mathrm{f} / \mathrm{fl}}$ mice as compared with WT mice at 7 d.p.i. (Figure 3E). While NK1.1 ${ }^{+}$ CD11 $b^{\text {int }}$ NK cells were increased 2-fold in the airspace of WT mice, NK cell numbers were significantly reduced in the lungs of SPC ${ }^{\mathrm{Cre}}$ / HOIL- $1 L^{\mathrm{f} / \mathrm{l} / \mathrm{l}}$ mice at the same time points (Figure $3 \mathrm{~F}$ ). Additionally, numbers of tissue-resident Siglec $F^{\text {hi }}$ CD $11 c^{\text {hi }}$ alveolar macrophages and $\mathrm{Ly} 6 \mathrm{G}^{+} \mathrm{CD} 11 \mathrm{~b}^{\mathrm{hi}} \mathrm{CD} 24^{\text {hi }}$ neutrophils during IAV infection were similar in WT and SPC Cre $/$ HOIL-1L $\mathrm{L}^{\mathrm{f} / \mathrm{l}}$ mice (Supplemental Figure $2, \mathrm{~L}$ and $\mathrm{M}$ ). While the innate response is important to limit viral spread, clearance of IAV from an infected host is dependent on the adaptive response $(37,38)$. Analysis of lung lymphoid populations by flow cytometry (Supplemental Figure 3B) after IAV infection showed a significant increase in the number of CD $44^{+} \mathrm{CD} 62 \mathrm{~L}^{-} \mathrm{CD} 8^{+}$ effector T cells and of $\mathrm{CD}^{+}{ }^{+} \mathrm{CD} 25^{+} \mathrm{Foxp}^{+}$Tregs in infected SPC Cre / HOIL-1L ${ }^{\mathrm{f} / \mathrm{fl}}$ mice compared with WT mice (Figure 3, G and $\mathrm{H}$ ). In contrast, no differences were detected in the number of $\mathrm{CD} 44^{+}$ CD62L ${ }^{-} \mathrm{CD} 4^{+} \mathrm{T}$ cells between WT and SPC Cre $/ \mathrm{HOIL}-1 \mathrm{~L}^{\mathrm{f} / \mathrm{fl}}$ mice after infection (Supplemental Figure 2N). Taken together, these data suggest that LUBAC-dependent signaling at the epithelial level impacts multiple facets of the host immune response during IAV infection.

To determine the impact of the decrease in viral titers (Figure 2D) on the protection from lung injury and decreased inflamma- tion in IAV-infected SPC Cre $/$ HOIL- $1 \mathrm{~L}^{\mathrm{fl} / \mathrm{fl}}$ mice, we administered a higher dose of WSN (WSN ${ }^{\text {Higher }}$, which resulted in viral titers at peak viremia comparable to those in WT mice infected with the standard lethal dose of WSN (WSN ${ }^{\text {StdL }}$ ) used in our in vivo model (Supplemental Figure 4A). SPC Cre $/$ HOIL-1L $1 \mathrm{~L}^{\mathrm{fl} / \mathrm{l}}$ mice that received $\mathrm{WSN}^{\text {Higher }}$ continued to display significantly reduced alveolar permeability, cellular infiltration, and IL-6 levels in BALF as compared with WT mice that received $\mathrm{WSN}^{\mathrm{StdL}}$ (Supplemental Figure $4, \mathrm{~B}-\mathrm{D})$. We observed no significant differences in BALF IFN- $\beta$ levels between $\mathrm{WSN}^{\text {StdL }}$-treated WT mice and $\mathrm{WSN}^{\text {Higher }}$-treated SPC ${ }^{\mathrm{Cre}} / \mathrm{HOIL}-1 \mathrm{~L}^{\mathrm{fl} / \mathrm{fl}}$ mice (Supplemental Figure 4E). These data suggest that decreased viral load does not drive the amelioration of lung injury in $\mathrm{SPC}^{\mathrm{Cre}} / \mathrm{HOIL}-1 \mathrm{~L}^{\mathrm{fl} / \mathrm{l}}$ mice.

IAV infection increases HOIL-1L in AECs. Analysis of the effect of IAV infection on the expression of LUBAC components revealed a significant upregulation of HOIL-1L at both the mRNA and the protein level by 3 d.p.i. through 7 d.p.i. (Figure 4, A and B) in AT2 cells isolated from WT mice infected with WSN. No differences in HOIP or SHARPIN expression were detected (Supplemental Figure 5, A-D). Similarly, when A549 cells were infected with 1 MOI WSN for $0,4,8$, and 16 hours, we measured a 5-fold increase in HOIL-1L mRNA and a 2-fold increase in HOIL-1L protein abundance by 16 hours postinfection (h.p.i.) (Supplemental Figure 5, E and $\mathrm{F}$ ), again, with no changes in expression of the other LUBAC components (Supplemental Figure 5, G-J).

In addition to its role in stabilizing LUBAC to promote maximal activity of the complex $(21,27)$, it has been described that 

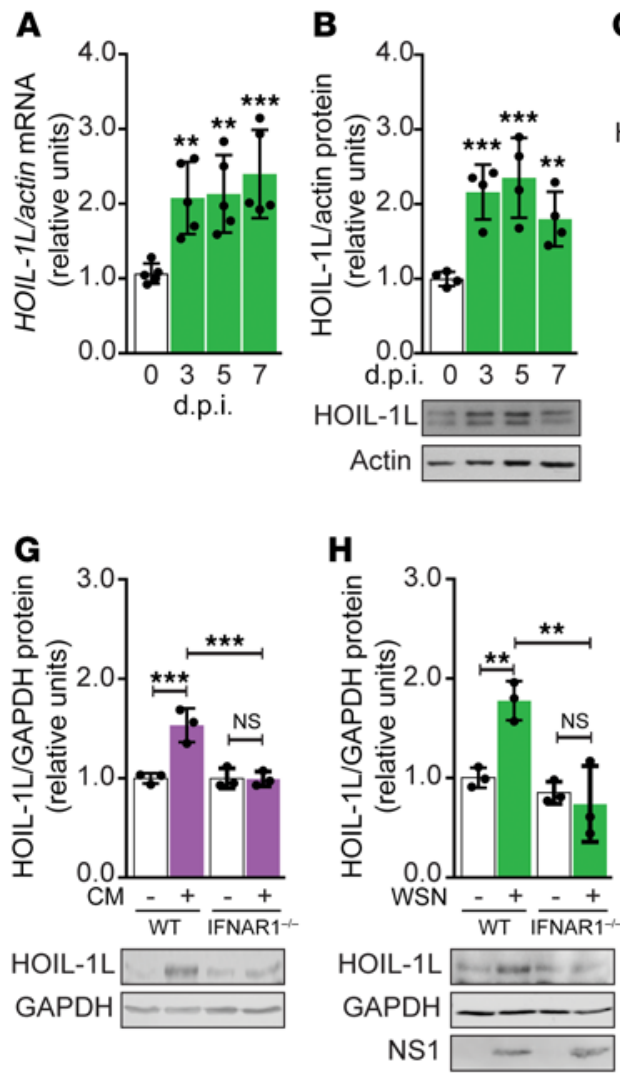
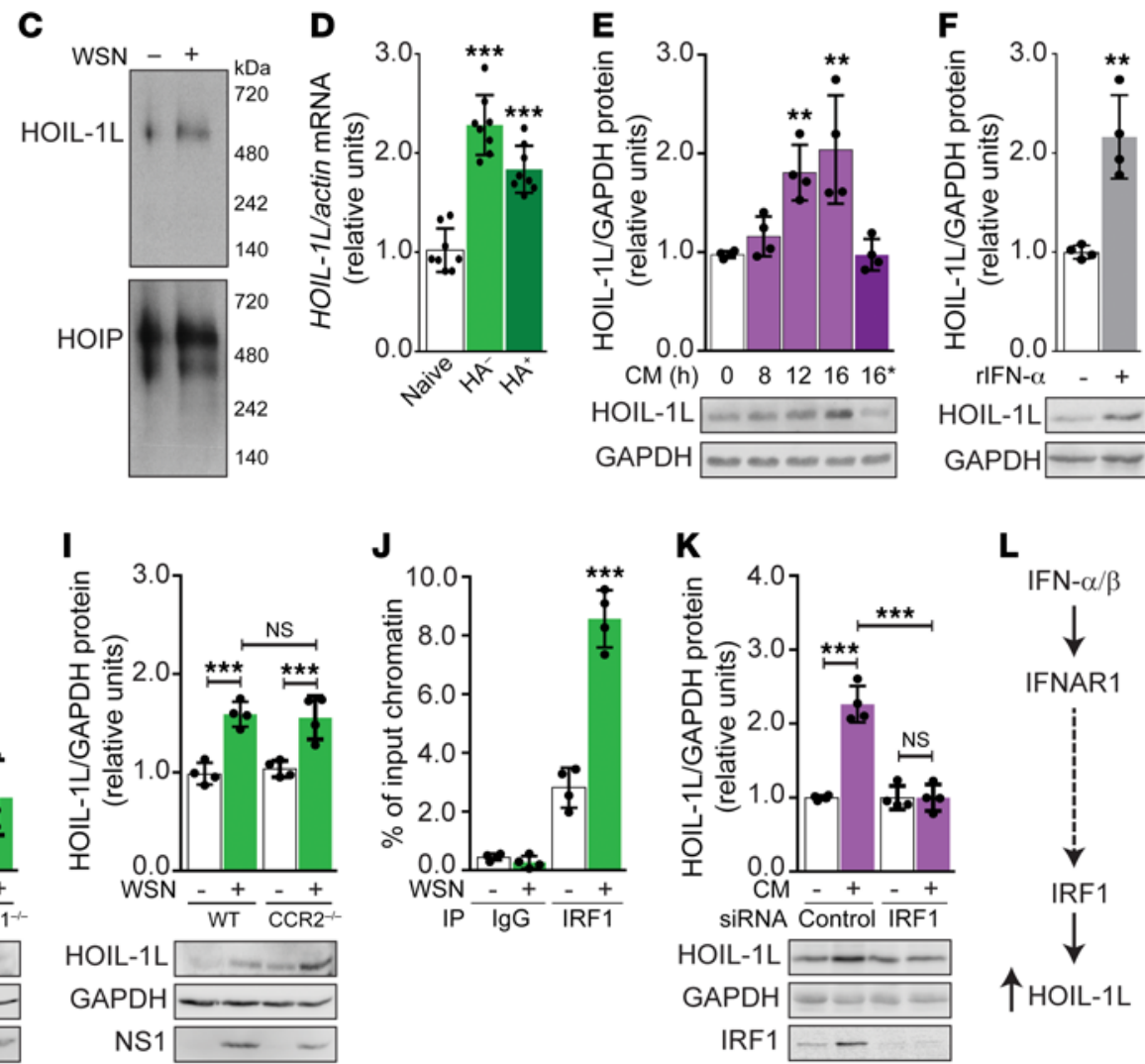

$\mathbf{L}$
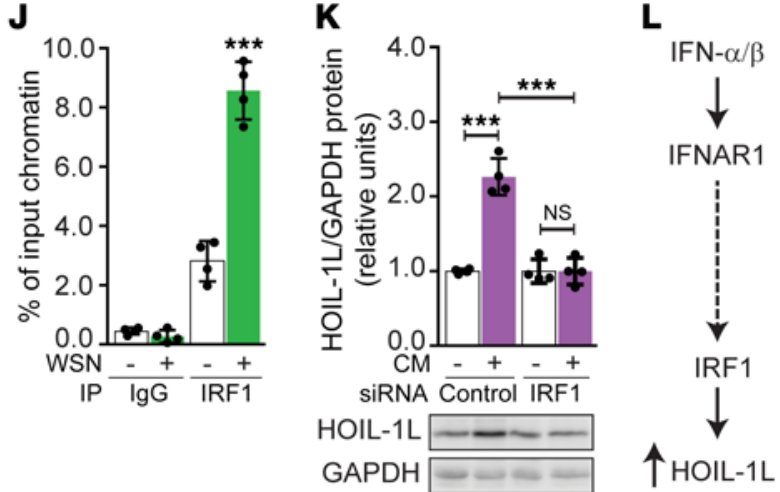

Figure 4. HOIL-1L is upregulated during IAV infection through a type I IFN receptor signaling axis. (A and B) AT2 cells were isolated from WT mice at 0,3 , 5, and 7 d.p.i. (A) HOIL-1L mRNA $(n=5)$. (B) Representative HOIL-1L immunoblot and its quantification $(n=4)$. (C) Representative native PACE immunoblot of HOIL-1L and HOIP expression in AT2 cells infected in vitro with WSN $(n=3)$. (D) HOIL-1L mRNA expression in AT2 cells from WT mice 0 and 3 d.p.i. sorted based on expression of the viral protein HA $(n=8)$. (E-I) Representative HOIL-1L immunoblots and quantification. (E) A549 cells treated for $0,8,12$, and 16 hours with conditioned medium (CM; "16*" indicates boiled CM) $(n=4)$. (F) A549 cells treated with recombinant IFN- $\alpha(n=4)$. (C) AT2 cells isolated from WT and IFNAR1 $1^{-1-}$ mice treated in vitro with CM $(n=3)$. (H) AT2 cells isolated from WT and IFNAR1 $1^{-1-}$ mice treated in vitro with WSN $(n=3)$. (I) AT2 cells isolated from WT and CCR2 $2^{-1-}$ mice treated in vitro with WSN $(n=4)$. (J) Quantitative reverse transcriptase PCR quantification of HOIL-1L promoter after ChIP of IRF1 in A549 cells $(n=4)$. (K) Representative HOIL-1L immunoblot and quantification in siControl- or silRF1-transfected A549 cells treated with CM $(n=4)$. (L) Proposed type I IFN pathway leading to HOIL-1L upregulation. Means \pm SD overlaid with individual data points representing replicates are depicted; ${ }^{* *} P<0.01,{ }^{* *} P<0.005$ (1-way ANOVA, Bonferroni post hoc test).

HOIL-1L has several LUBAC-independent functions, such as acting as an E3 ubiquitin ligase to target proteins such as PKCל for degradation as seen in adaptation to hypoxia and cancer $(23$, $31,39)$. Nondenaturing native gel electrophoresis of lysates from WT AT2 cells or A549 cells exposed to WSN in vitro revealed that HOIL-1L runs consistent with the $600 \mathrm{kDa}$ molecular weight of LUBAC (25) (Figure 4C and Supplemental Figure 5K). These results suggest that during IAV infection newly synthesized HOIL$1 \mathrm{~L}$ is incorporated into LUBAC.

IAV infection upregulates HOIL-1L via autocrine and paracrine type I IFN signaling. It has been described that virus-infected epithelial cells signal to adjacent noninfected cells through the release of mediators, such as lipids, cytokines, and IFNs (12, 40, 41). We sought to investigate whether the increase in HOIL-1L expression was a direct consequence of viral infection, a response to secreted autocrine and paracrine mediators, or both. At 0 and 3 d.p.i., single-cell whole-lung suspensions of WT AT2 cells, defined as CD45-CD31-EpCAM ${ }^{+}$, were FACS-sorted based on the presence of the viral surface protein HA to separate infected $\left(\mathrm{HA}^{+}\right)$from noninfected $\left(\mathrm{HA}^{-}\right)$AT2 cell populations (Supplemen- tal Figure 3C). Infection status of each population was confirmed by quantitative reverse transcriptase PCR expression of IAV nucleoprotein (NP; a viral genome-associated protein necessary for replication in target cells) (Supplemental Figure 5L and ref. 42). Analysis of mRNA from $\mathrm{HA}^{-}$and $\mathrm{HA}^{+}$populations showed that infection with IAV was not required for HOIL-1L upregulation, as $H O I L-1 L$ mRNA was equally upregulated in both $\mathrm{HA}^{-}$and $\mathrm{HA}^{+}$ populations (Figure $4 \mathrm{D}$ ). These results were recapitulated in vitro, where UV-irradiated conditioned medium (CM) from untreated $\left(\mathrm{CM}^{-}\right)$or IAV-infected $\left(\mathrm{CM}^{+}\right)$A549 cells was added to naive cells. In response to $\mathrm{CM}^{+}$, a 2-fold induction of HOIL-1L was detected as early as 12 h.p.i., which was inhibited by heat denaturation of protein mediators within $\mathrm{CM}^{+}$(Figure 4E). Taken together, these results suggest that a protein mediator released from IAV-infected epithelial cells is sufficient to upregulate HOIL-1L in an autocrine or paracrine manner.

We next sought to identify the cytokine(s) necessary for the observed autocrine/paracrine regulation of HOIL-1L. It has been reported that IFN- $\alpha$ and IFN- $\gamma$ can regulate the expression of LUBAC components in SHARPIN-deficient mice (43). In our mod- 

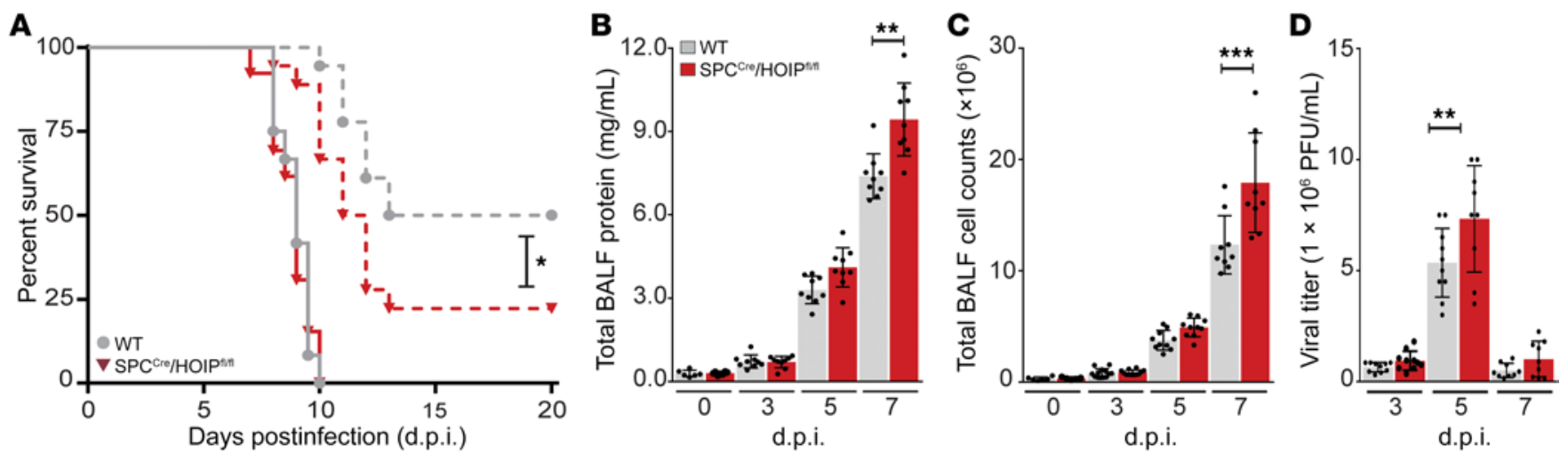

Figure 5. Loss of HOIP from the alveolar epithelium decreases survival and enhances lung injury in mice infected with IAV. (A) Survival of WT and $\mathrm{SPC}^{\mathrm{Cre}} / \mathrm{HOIP}^{\mathrm{fl} / \mathrm{fl}}$ mice infected with a low $(n=18$, dashed line) or lethal $(n=13$, solid line) dose of WSN. (B and C) BALF was analyzed at 0, 3, 5, and 7 d.p.i. $(n=9)$ for total protein concentrations (B) and cellular infiltration (C). (D) Viral titers in WT and SPC Cre $/$ HOIPfl/fl mice at 3, 5, and 7 d.p.i. ( $n=9$ ). Means \pm SD overlaid with individual data points representing replicates are depicted; ${ }^{*} P<0.05,{ }^{* *} P<0.01,{ }^{* *} P<0.005$ (A, 2-tailed log-rank Mantel-Cox test; B-D, 1-way ANOVA, Bonferroni post hoc test).

el, increased type I IFN (IFN- $\alpha$, IFN- $\beta$ ) production in vivo paralleled HOIL-1L upregulation (Figure 3, B and C). Thus, we tested whether IFN- $\alpha$ was sufficient to increase HOIL-1L expression by treating A549 cells with recombinant human IFN- $\alpha$, and found significantly increased HOIL-1L expression in A549 cell lysates (Figure 4F). Additionally, AT2 cells isolated from type I IFN receptordeficient (IFNAR1-deficient; IFNAR1 ${ }^{-/}$) mice exposed to $\mathrm{CM}^{+}$ failed to upregulate HOIL-1L as compared with WT AT2 cells (Figure $4 \mathrm{G}$ ). We obtained similar results with in vitro siRNA silencing of IFNAR1 compared with siControl-transfected A549 cells treated with $\mathrm{CM}^{+}$(Supplemental Figure $5 \mathrm{M}$ ). To investigate whether direct viral infection was sufficient to induce HOIL-1L expression independent of IFNAR1 signaling, AT2 cells from IFNAR1 $1^{-/}$mice were infected with WSN. IFNAR1 deficiency inhibited HOIL-1L upregulation in comparison with WSN-infected WT AT2 cells, despite active virus replication as measured by expression of the IAV nonstructural protein 1 (NS1) (Supplemental Figure $5 \mathrm{~N}$ ). In vitro silencing of IFNAR1 yielded similar results, with inhibition of HOIL-1L upregulation in comparison with siControl A549 cells treated with WSN (Figure 4H). Conversely, upon $\mathrm{CM}^{+}$(Supplemental Figure $5 \mathrm{O}$ ) or WSN treatment (Figure 4I), AT2 cells isolated from mice lacking the receptor for CCL-2/MCP-1 (CCR2) upregulated HOIL$1 \mathrm{~L}$ expression comparably with WT AT 2 cells. These results suggest that signaling downstream of IFNAR1 is necessary to induce HOIL$1 \mathrm{~L}$ expression in both human and mouse AECs.

We sought to identify the transcription factor(s) regulating HOIL1L downstream of IFNAR1. IFN regulatory factors (IRF1-IRF9) bind to variations of the IFN-stimulated response element consensus sequence $(44,45)$ found within the HOIL-1L promoter (43). In silico analysis (46) of the human and mouse HOIL-1L promoters identified a shared putative binding site for IRF1. To validate a direct role of IRF1 in HOIL-1L regulation, we performed a ChIP assay, revealing a 5 -fold increase in the direct binding of IRF1 to the HOIL-1L promoter during WSN infection of A549 cells (Figure 4J). Additionally, in vitro silencing of IRF1 inhibited HOIL-1L upregulation in response to $\mathrm{CM}^{+}$(Figure 4K). Collectively, the data suggest that IRF1 binds to the HOIL-1L promoter and regulates HOIL-1L induction in response to influenza-induced type I IFN signaling (Figure $4 \mathrm{~L}$ ).
Deletion of epithelial HOIP during IAV infection worsens lung injury and decreases survival. To further assess the role of LUBAC in regulating the epithelial-driven immune response to IAV, we generated a transgenic mouse with HOIP excised specifically from the lung epithelium (SPC ${ }^{\mathrm{Cre}} / \mathrm{HOIP}^{\mathrm{f} / \mathrm{fl}}$ ) as described in Methods. Loss of HOIP protein levels, as well as the presence of HOIL-1L and SHARPIN, was confirmed by immunoblot of WT and SPC Cre / HOIP ${ }^{\mathrm{fl} / \mathrm{fl}}$ AT2 cell lysates (Supplemental Figure 6A). Administration of low-dose WSN to SPC Cre/HOIPf//l mice significantly decreased survival in comparison with WT mice with a mean survival of 11.5 and 13 d.p.i., respectively (Figure 5A). Additionally, careful examination of $\mathrm{H} \& \mathrm{E}$-stained lung sections showed enhanced severity of lung injury in $\mathrm{SPC}^{\mathrm{Cre}} / \mathrm{HOIP}^{\mathrm{f} / \mathrm{fl}}$ mice as compared with WT at 7 d.p.i. (Supplemental Figure 6, B and C). The lethal dose of WSN resulted in $100 \%$ mortality in both WT and $\mathrm{SPC}^{\mathrm{Cre}} / \mathrm{HOIP}^{\mathrm{f} / \mathrm{fl}}$ mice by 10 d.p.i.; however, at earlier time points we observed differences in markers of lung injury, i.e., increased BALF protein (Figure 5B) and cell count (Figure 5C). Analysis of whole lung by plaque assay revealed an increase in viral load in SPC $\mathrm{Sre}^{\mathrm{C}} / \mathrm{HOIP}^{\mathrm{fl} / \mathrm{fl}}$ mice as compared with WT mice at peak viremia 5 d.p.i. with no significant differences in viral clearance by 7 d.p.i. (Figure 5D). Consistent with our observations that loss of alveolar epithelial HOIP expression is detrimental in the response to IAV infection, data mined from a previously reported genome-wide association study data set of 232 African American patients with ARDS and 162 at-risk control subjects (47) revealed an SNP in the HOIP gene (RNF31) that coincides with worsened ARDS prognosis (Supplemental Figure 7). The identified SNP results in an amino acid change from valine to isoleucine (V1061I) between the RING2 and LDD domains of HOIP that make up the "catalytic core" $(22,27)$. Together these results support our findings in SPCCre/ HOIL-1 $\mathrm{L}^{\mathrm{f} / \mathrm{fl}}$ mice that a degree of LUBAC activity is protective during inflammatory lung injury, as inhibition of LUBAC in SPC Cre / $\mathrm{HOIP}^{\mathrm{f} / \mathrm{fl}}$ mice results in greater morbidity and mortality.

AT2 cells isolated from SPC $\mathrm{Sre}^{\mathrm{C}} / \mathrm{HOIP}^{\mathrm{f} / \mathrm{fl}}$ mice at 0 and 4 d.p.i. have a markedly impaired NF- $\kappa \mathrm{B}$ response as shown by significantly decreased mRNA encoding $M c p 1, I l 6$, and Ifnb compared with AT2 cells isolated from WT mice (Figure 6, A-C). Howev- 
A

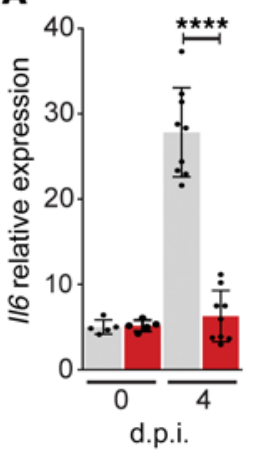

B

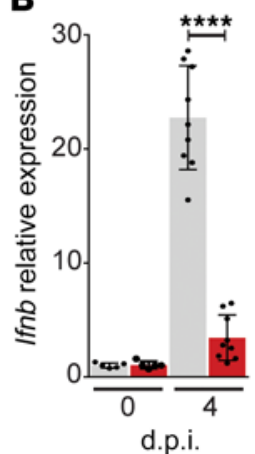

C

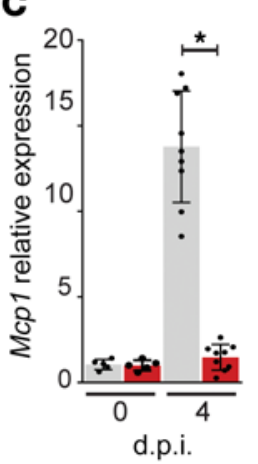

D

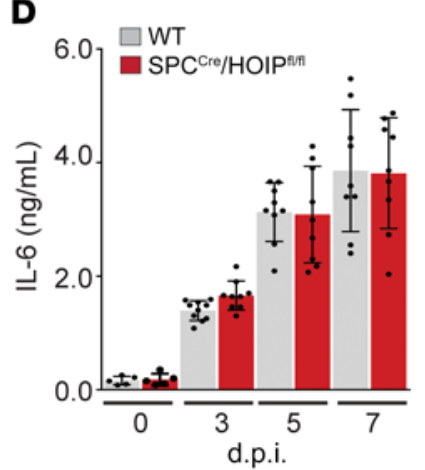

H

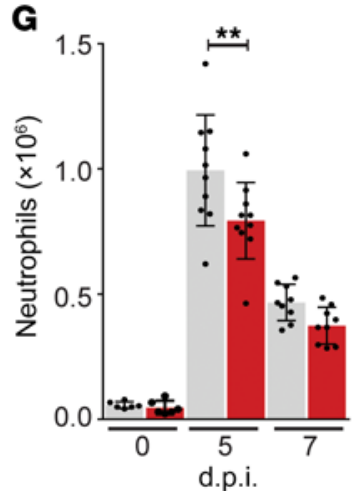

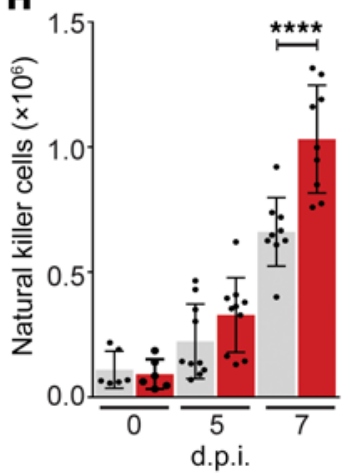

E

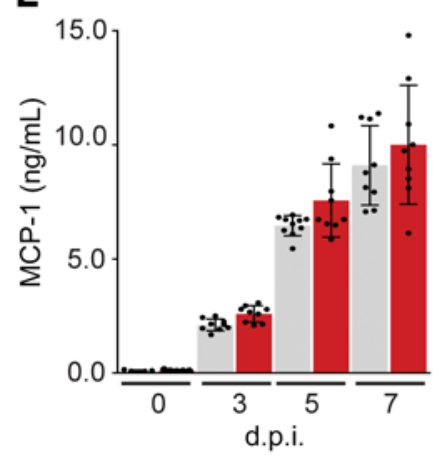

I

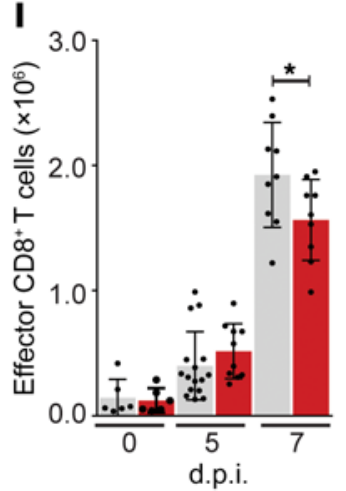

Figure 6. Loss of HOIP from the alveolar epithelium inhibits alveolar epithelial-driven inflammatory response to IAV infection. (A-C) WT and SPCCre/ HOIPfl/fl mice were infected with a lethal dose of WSN. (A-C) AT2 cells at 0 and 4 d.p.i. $(n=9)$ analyzed for $/ 16$ mRNA (A), Ifnb mRNA (B), and Mcp1 mRNA (C). (D-F) BALF analyzed by ELISA at 0, 3, 5, and 7 d.p.i. $(n=9)$ for IL-6 (D), MCP-1 (E), and IFN- $\beta(\mathbf{F})$. (G-I) Lung immune cell populations at 0 , 5 , and 7 ( $n=9$ ) d.p.i. analyzed by flow cytometry for Ly6C+CD11b+CD24+ neutrophils (G), NK1.1+CD11b hi CD24 hi natural killer cells (H), and CD44+CD62L-CD8 ${ }^{+} T$ cells $(\mathbf{I})$. Means \pm SD overlaid with individual data points representing replicates are depicted; ${ }^{*} P<0.05,{ }^{* *} P<0.01,{ }^{* * *} P<0.0001$ (1-way ANOVA, Bonferroni post hoc test).

er, analysis of BALF revealed similar levels of IL-6 and MCP-1 between WT and $\mathrm{SPC}^{\mathrm{Cre}} / \mathrm{HOIP}^{\mathrm{fl} / \mathrm{fl}}$ mice over the course of infection (Figure 6, D and E), while IFN- $\beta$ levels were dampened in $\mathrm{SPC}^{\mathrm{Cre}}$ / $\mathrm{HOIP}^{\mathrm{fl} / \mathrm{fl}}$ mice compared with WT mice at 5 d.p.i. (Figure $6 \mathrm{~F}$ ). Analysis of lung myeloid and lymphoid populations by flow cytometry at 0,5 , and 7 d.p.i. revealed similar recruitment of immune cell populations in WT and SPC ${ }^{\mathrm{Cre}} / \mathrm{HOIP}{ }^{\mathrm{fl} / \mathrm{fl}}$ mice (Figure 6, G and $\mathrm{H}$, and Supplemental Figure 6, D-F). We detected similar numbers of SiglecF $F^{\text {hi }} \mathrm{CD} 11 \mathrm{c}^{\text {hi }}$ alveolar macrophages (Supplemental Figure 6D), CD $11 b^{\text {hi }} \mathrm{MHCII}^{\text {hi }} \mathrm{CD} 24^{\text {lo }} \mathrm{CD} 64^{\text {hi }}$ monocyte-derived inflammatory macrophages (Supplemental Figure 6E), and CD11b ${ }^{\text {hi }}$ MHCII $^{\mathrm{lo}}$ Ly6C ${ }^{\text {hi }}$ classical monocytes (Supplemental Figure 6F) in $\mathrm{WT}$ and $\mathrm{SPC}^{\mathrm{Cre}} / \mathrm{HOIP}^{\mathrm{fl} / \mathrm{fl}}$ mice. In contrast, we observed a significant reduction in neutrophil recruitment in $\mathrm{SPC}^{\mathrm{Cre}} / \mathrm{HOIP}^{\mathrm{fl} / \mathrm{fl}}$ mice as compared with WT mice at 5 d.p.i. (Figure 6G) as well as a 2 -fold increase in the recruitment of $\mathrm{NK} 1.1^{+} \mathrm{CD} 11 b^{\text {int }} \mathrm{NK}$ cells in $\mathrm{SPC}^{\mathrm{Cre}} /$ $\mathrm{HOIP}^{\mathrm{fl} / \mathrm{fl}}$ mice as compared with WT mice at 7 d.p.i. (Figure $6 \mathrm{H}$ ). Within the lymphoid lineage we detected a significant reduction in the number of $\mathrm{CD} 44^{+} \mathrm{CD} 62 \mathrm{~L}^{-} \mathrm{CD}^{+} \mathrm{T}$ cells at 7 d.p.i. (Figure 6I) with no appreciable differences in the total number of either $\mathrm{CD} 4{ }^{+} \mathrm{CD} 25^{+} \mathrm{Foxp}^{+}$Tregs or CD $44^{+} \mathrm{CD} 62 \mathrm{~L}^{-} \mathrm{CD} 4^{+} \mathrm{T}$ cells in $\mathrm{SPC}^{\mathrm{Cre}} /$ $\mathrm{HOIP}^{\mathrm{fl} / \mathrm{fl}}$ mice compared with WT mice (Supplemental Figure 6, $\mathrm{G}$ and $\mathrm{H})$. Together these results suggest that a certain amount of LUBAC activity at the level of the lung epithelium is necessary to coordinate the proper host response to IAV infection and confer protection from lung injury.
Restoration of $N F-\kappa B$ signaling in $S P C^{C r e} / H O I P^{f / f l}$ mice reduces lung injury and improves survival during IAV infection. LUBAC is necessary for robust NF- $\mathrm{KB}$ activation, as linear ubiquitin chains on NEMO provide a stable docking site for additional IKK complexes, facilitating efficient activation of IKK $\alpha$ and IKK $\beta$, and phosphorylation and degradation of $\mathrm{I} \kappa \mathrm{B} \alpha(21,28,29)$. To confirm that loss of LUBAC-dependent NF- $\mathrm{BB}$ signaling in AT2 cells was driving mortality in IAV-infected SPC ${ }^{\mathrm{Cre}} / \mathrm{HOIP}^{\mathrm{f} / \mathrm{l} \mathrm{l}}$ mice, we used an adenovirus coding for the constitutively active form of IKK $\beta$ (AdIKK $\beta$-CA) (48), the primary kinase responsible for phosphorylation of IкB $\alpha$ (49). SPC ${ }^{\mathrm{Cre}} / \mathrm{HOIP}^{\mathrm{f} / \mathrm{fl}}$ mice were instilled with a noncoding adenovirus (AdNull) or AdIKK $\beta$-CA, 14 days before infection with WSN (Supplemental Figure 8A). Expression of IKK $\beta$ was confirmed by immunoblot of isolated AT2 cell lysates (Figure 7B). While no difference in the content of IL- 6 or IFN- $\beta$ levels in BALF was observed between naive and AdNull-treated SPC ${ }^{\mathrm{Cre}} / \mathrm{HOIP}^{\mathrm{f} / \mathrm{fl}}$ mice, IKK $\beta-\mathrm{CA}$ was sufficient to induce their production (Supplemental Figure 8, B and $C$ ). Taken together, these results suggest that overexpression of IKK $\beta$-CA in SPC ${ }^{\text {Cre }} / \mathrm{HOIP}^{\mathrm{f} / \mathrm{l} \text { l }}$ mice is sufficient to restore epithelialdriven NF- $\kappa$ B signaling downstream of LUBAC.

We next sought to determine whether restoration of NF- $\mathrm{BB}$ signaling downstream of LUBAC in SPC ${ }^{\mathrm{Cre}} / \mathrm{HOIP}^{\mathrm{fl} / \mathrm{fl}}$ mice would impact survival during IAV infection. Low-dose WSN infection resulted in $100 \%$ mortality by 15 d.p.i. in AdNull-treated SPC Cre/ HOIP $^{\mathrm{fl} / \mathrm{fl}}$ mice. However, AdIKK $\beta$-CA-treated SPC $\mathrm{Cre}^{\mathrm{Cr}} / \mathrm{HOIP}^{\mathrm{f} / \mathrm{fl}}$ mice were significantly protected, with over $50 \%$ survival at the same 

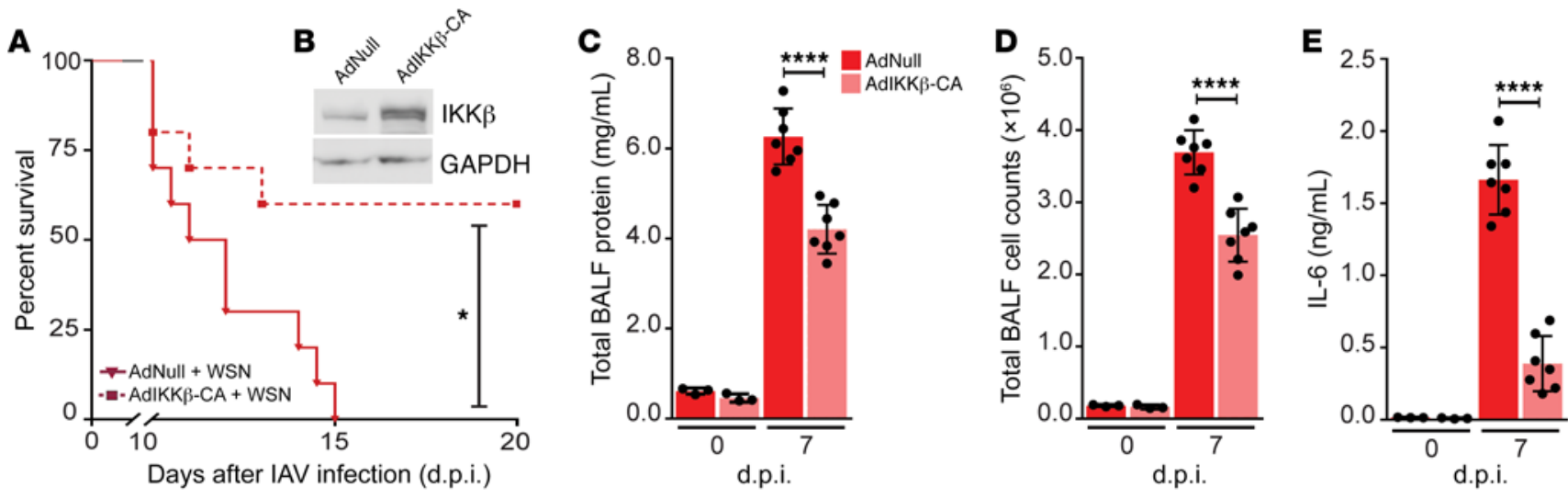

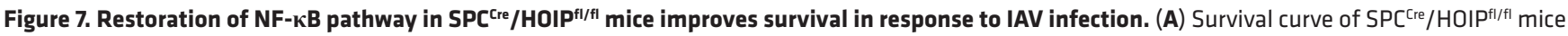
given either AdNull ( $n=11$, solid line) or AdIKK $\beta$-CA ( $n=10$, dashed line) followed by low-dose WSN. (B) Immunoblot of AT2 cell lysates showing overexpression of IKK $\beta(n=3)$. (C-E) BALF from SPC Cre $/ H O I P^{f / / f l}$ mice given either AdNull or AdIKK $\beta$-CA collected at $0(n=3)$ and 7 d.p.i. $(n=7)$ and low-dose WSN and analyzed for total protein concentration (C), cellular infiltration (D), and IL-6 (E). Means \pm SD overlaid with individual data points representing replicates are depicted; ${ }^{*} P<0.05,{ }^{* * *} P<0.0001$ (A, 2-tailed log-rank Mantel-Cox test; C-E, 1-way ANOVA, Bonferroni post hoc test).

time point (Figure 7A). Consistent with improved survival, we observed significant reductions in protein levels, infiltrating cell counts, and IL-6 in the BALF of SPC Cre/HOIP ${ }^{\mathrm{A} / \mathrm{l}}$ mice given AdIK$\mathrm{K} \beta$-CA as compared with AdNull treatment prior to WSN infection (Figure 7, C-E). Experiments were performed 7 days after WSN infection, a time at which both AdNull- and AdIKK $\beta$-CA-treated $\mathrm{SPC}^{\mathrm{Cre}} / \mathrm{HOIP}^{\mathrm{A} / \mathrm{fl}}$ mice had lost a significant percentage of body weight (data not shown), but before any mortality events occurred (Figure 7A). Collectively, the data suggest that LUBAC-mediated NF- $\mathrm{KB}$ activation in lung epithelial cells is necessary for orchestration of a sufficient host response during IAV infection.

\section{Discussion}

IAV infection can cause severe pneumonia, respiratory failure, and death $(2,4)$. A heterogeneous response to IAV with the same virulence exists within the population, suggesting that host factors play a crucial role regulating the inflammatory response and determining the severity of lung injury (2-4). While current anti-influenza strategies are limited to yearly vaccination or administration of antiviral drugs, it is clear that inclusion of strategies that target the host pathways will be beneficial $(7,50)$. Here we report that the level of LUBAC activity in pulmonary epithelial cells is a critical determinant of the NF- $\mathrm{KB}$ inflammatory response to IAV infection. Tight regulation of NF- $\mathrm{kB}$ is critical for the maintenance of immune homeostasis, as its uncontrolled activation may result in "cytokine storm" with severe pathological consequences (13-17). During IAV infection, expression of the LUBAC component HOIL$1 \mathrm{~L}$ is upregulated in AECs by a mechanism that involves direct binding of IRF1 to the HOIL-1L promoter in response to type I IFNs (Figure 8). This upregulation of HOIL-1L during IAV infection appears to be maladaptive, as attenuation of LUBAC activity by silencing of full-length HOIL-1L in the lung epithelium is protective. In contrast, we show that complete loss of LUBAC activity, via deletion of HOIP in lung epithelial cells, leads to impaired epithelial-driven immune response and increased mortality. In support of these observations, an SNP predicted to result in an amino acid change in the "catalytic core" of HOIP was associated with a worse prognosis in a cohort of African American patients with ARDS compared with controls. These findings highlight the fine line between an excessive and an inadequate immune response and suggest that therapeutic modulation of LUBAC activity may be crucial, as it functions as a rheostat regulating the amplitude of the host response to IAV infection.

While several studies have focused on LUBAC signaling downstream of TNFRSF and the IL- $1 \beta$ receptor, changes in the expression of LUBAC components in these signaling pathways have not been reported (20-26, 34, 35, 51). In contrast, we observed that during IAV infection, HOIL-1L expression is increased and associates with the other LUBAC components. We found that the binding of IRF1 directly to the HOIL-1L promoter downstream of IFNAR1 is necessary for HOIL-1L upregulation during IAV infection in both human and mouse AECs. While we found type I IFN signaling to be necessary, we cannot discount the possible combinatorial role of additional factors, as IRF1 has also been shown to be upregulated in response to IFN- $\gamma$ and TNF- $\alpha$ stimulation (43, $52,53)$. We detected very low levels of TNF- $\alpha$ in BALF in response to IAV infection, suggesting a minor, if any, role for TNF- $\alpha$ signaling during in vivo HOIL-1L upregulation. IFN- $\gamma$ plays an important role in the antiviral response and has a robust effect on IRF1 induction $(43,53)$, and while we do not observe an increase in IFN- $\gamma$ until 5 d.p.i., it may contribute to maintaining elevated levels of HOIL$1 \mathrm{~L}$ during IAV infection. The molecular mechanism responsible for the increased levels of HOIL-1L during IAV infection may be of great interest, as it may contribute to increased LUBAC stability and the exaggerated production of cytokines during IAV infection.

Expression of type I IFN early during infection restricts viral spread, both in the infected cell where IFN-stimulated gene products directly target hijacked viral pathways, and in uninfected cells by priming an antiviral state $(17,54)$. Due to the range of pathogenicity among IAV strains, divergent reports exist on the role of type I IFN signaling in limiting mortality during IAV infection $(11,55)$. However, a direct correlation between IAV-induced high IFN- $\alpha / \beta$ levels and high morbidity and mortality has been reported $(11,54,56,57)$. As the infection persists, an exuberant and sus- 


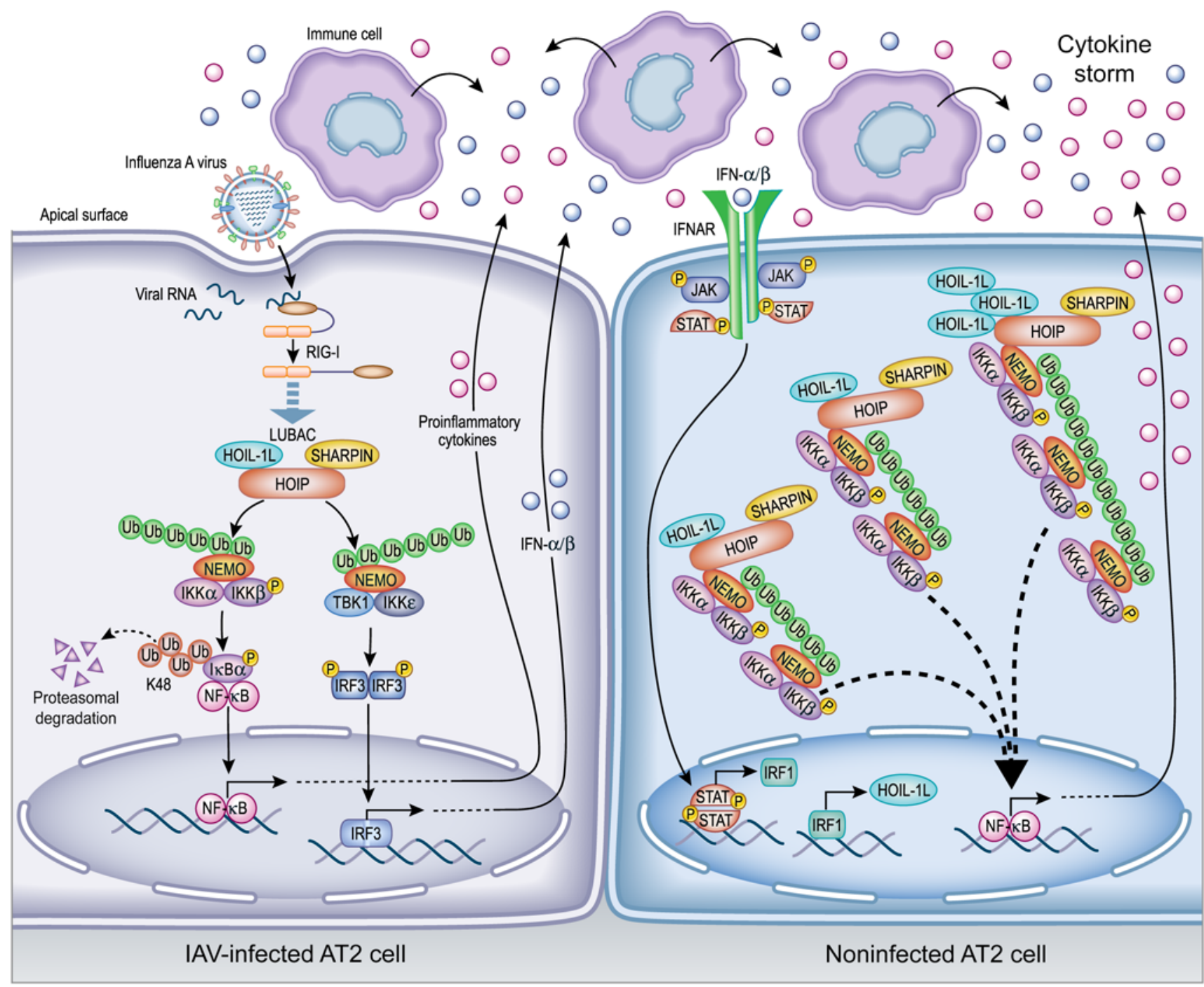

Figure 8. Illustration representing HOIL-1L upregulation via IRF1 in AT2 cells. Upon IAV infection, LUBAC-dependent activation of NF- $K B$ and IRF3 pathways occurs downstream of RIG-I. Subsequent release of proinflammatory cytokines and type I IFNs recruits immune cells to the airspace, contributing to the growing cytokine storm. In noninfected AT2 cells, IFN secreted by either epithelial cells or the recruited immune cells binds to IFNAR1, triggering a signal cascade that upregulates HOIL-1L via IRF1. Newly synthesized HOIL-1L may contribute to either an increased number of LUBAC complexes or a change in LUBAC stoichiometry to include a higher ratio of HOIL-1L within the complex. Both situations result in increased stability of LUBAC and exaggerated production of cytokines, which contributes to the morbidity and mortality observed in severe cases of influenza infection.

tained IFN response can have deleterious effects by enhancing TRAIL-mediated cell death (11) or facilitating secondary bacterial infections (57). Thus, we propose that the IFN-mediated regulation of HOIL-1L is a host maladaptation to IAV infection, as we observe significant protection in SPC Cre $/$ HOIL-1L ${ }^{\mathrm{f} / \mathrm{fl}}$ mice. While HOIL-1L does not directly contribute to linear ubiquitin chain formation $(23,27)$, its binding within LUBAC masks a ubiquitination site on HOIP to prevent its proteasomal degradation and releases HOIP's autoinhibitory fold to maximize LUBAC activity $(23,26$, 27, 32). Thus, increased abundance of HOIL-1L may contribute to the enhanced stability and activity of LUBAC. As the stoichiometry of LUBAC has not been elucidated $(23,26,27)$, increased LUBAC-associated HOIL-1L may represent an increase in the ratio of HOIL-1L within the complex or an increase in the abundance of LUBAC complexes, either of which may contribute to enhanced NEMO linear ubiquitination and robust downstream $\mathrm{NF}-\kappa \mathrm{B}$ activation (Figure 8 ).

Loss of LUBAC activity has been shown to have cell typespecific effects $(23,58-60)$. For example, patients with LUBAC deficiency present with both autoinflammation and immunodeficiency. In mice, global HOIP- and HOIL-1L-null mice are embryonically lethal, while global SHARPIN deficiency results in viability with multiorgan inflammation. In contrast, HOIL-1L $\mathrm{L}^{-/-}$ mice, which express a low-level N-terminus HOIL-1L (N-HOIL1L) that retains the UBL and LTM domains for heterotrimeric LUBAC formation (33), are viable with no overt phenotype. To circumvent confounding cell type-specific phenotypes in vivo, we used a pulmonary epithelial cell-specific transgenic mouse model (SPC $\left.{ }^{\mathrm{Cre}}\right) . \mathrm{SPC}^{\mathrm{Cre}} / \mathrm{HOIL}-1 \mathrm{~L}^{\mathrm{fl} / \mathrm{fl}}$ mice lack full-length HOIL-1L and express a low level N-HOIL-1L, and thus are capable of a certain 
level of LUBAC-dependent signaling. While it has been reported that influenza infection of mice with chronic proliferative dermatitis due to global SHARPIN deficiency results in decreased survival in comparison with WT mice (61), here we have shown that IAV infection of phenotypically normal SPC ${ }^{\mathrm{Cre}} / \mathrm{HOIL}-1 \mathrm{~L}^{\mathrm{f} / \mathrm{fl}}$ mice resulted in increased survival in comparison with WT mice. While the mice used in this study have low expression of the truncated variant of HOIL-1L and may retain some LUBAC activity, SPC ${ }^{\mathrm{Cre}}$ / HOIL- $1 \mathrm{~L}^{\mathrm{f} / \mathrm{fl}}$ mice have significantly reduced cytokine and IFN levels in response to IAV infection. This is consistent with our in vitro data where silencing of full-length HOIL-1L reduced the linear ubiquitination of NEMO, activation of NF- $\kappa$ B and IRF3, and IL-6 and IFN- $\beta$ secretion. Additionally, mouse embryonic fibroblasts derived from HOIL-1L $\mathrm{L}^{-/-}$mice, which do not express full-length HOIL-1L but instead express the truncated variant (21), have reduced levels of 600-kDa LUBAC (32) as well as impaired NF- $\kappa B$ signaling (21). These findings suggest that deletion of full-length HOIL-1L and low expression of the truncated N-terminal fragment is sufficient to destabilize LUBAC, and reduce LUBAC-dependent inflammatory signaling. We find that fine tuning of LUBAC activity through deletion of lung epithelial full-length HOIL-1L is protective during IAV infection, reducing lung injury and viral titers and promoting survival.

The effects of lung epithelial HOIL-1L deletion on the innate and adaptive immune responses to IAV infection suggest that multiple mechanisms may be responsible for the lower viral titers, reduced injury, and improved survival in $\mathrm{SPC}^{\mathrm{Cre}} / \mathrm{HOIL}-1 \mathrm{~L}^{\mathrm{fl} / \mathrm{fl}}$ mice compared with WT mice. During IAV infection respiratory epithelial cells produce large amounts of MCP-1, resulting in the recruitment of classical monocytes and inflammatory monocyte-derived macrophages that produce large amounts of cytokines and are the predominant cause of immune pathology during influenza infection $(14,56)$. NK cells recruited to the lung also contribute to tissue damage as they target infected AECs for destruction, sacrificing barrier integrity in an effort to limit viral spread $(62,63)$. As such, reductions in inflammatory cytokines, classical monocytes, inflammatory monocyte-derived macrophages, and NK cells seen in IAV-infected SPC ${ }^{\mathrm{Cre}} / \mathrm{HOIL}-1 \mathrm{~L}^{\mathrm{f} / \mathrm{fl}}$ mice likely play a significant role in the protection from lung injury and increased survival during IAV infection. In addition, $\mathrm{CD}^{+} \mathrm{T}$ cells were increased in $\mathrm{SPC}^{\mathrm{Cre}} / \mathrm{HOIL}-1 \mathrm{~L}^{\mathrm{fl} / \mathrm{ll}}$ mice as compared with WT mice. The specific targeting of infected AECs by $\mathrm{CD}^{+} \mathrm{T}$ cells is the primary mode of viral clearance in the IAV-infected lung (64). While we show that the improved survival was not exclusively attributable to lower viral loads, as SPC ${ }^{\text {Cre }} / \mathrm{HOIL}-1 \mathrm{~L}^{\mathrm{f} / \mathrm{fl}}$ mice remain protected after infection with a higher titer of virus, increased antiviral CD8 ${ }^{+} \mathrm{T}$ cells in SPC ${ }^{\mathrm{Cre}} / \mathrm{HOIL}-1 \mathrm{~L}^{\mathrm{fl} / \mathrm{fl}}$ mice may contribute to the measured decrease in peak viral titers and contribute to potential long-term survival. In support of this, it has been reported that IAV infection of $\mathrm{T}$ cell-deficient mice resulted in a lower survival rate by 21 d.p.i., persistent lung injury, and higher viral titers in comparison with WT mice $(64,65)$. Moreover, there was a significant increase in the number of Tregs in SPC Cre $/$ HOIL- $1 \mathrm{~L}^{\mathrm{fl} / \mathrm{fl}}$ mice as compared with WT mice. Tregs contribute to quelling of the inflammatory response to limit tissue damage as well as to the promotion of tissue repair (66). Together with the concurrent increased levels of the antiinflammatory cytokine IL-10 in SPC Cre $/ \mathrm{HOIL}-1 \mathrm{~L}^{\mathrm{fl} / \mathrm{fl}}$ mice as compared with WT mice, our results suggest that reduced inflammation confers protection during IAV infection. This is supported by clinical findings in patients with worse outcomes of the pandemic 2009 H1N1 IAV who had elevated levels of circulating IL-6 despite viral clearance (15). These findings highlight the ability of LUBAC-dependent lung epithelial signaling to coordinate multiple components of the immune responses to IAV infection.

A balance between an excessive and an inadequate immune response to infection is necessary for optimal recovery from IAV infection. In contrast to SPC ${ }^{\text {Cre }} / \mathrm{HOIL}-1 \mathrm{~L}^{\mathrm{f} / \mathrm{l} / \mathrm{l}}$ mice, genetic deletion of HOIP in lung epithelial cells resulted in worse lung injury and decreased survival in response to IAV infection. These findings are in agreement with worse ARDS prognosis associated with an SNP within the "catalytic core" of HOIP, which may affect its activity or protein-protein interactions necessary for NF- $\kappa \mathrm{B}$ activation. AT2 cells from SPC ${ }^{\mathrm{Cre}} / \mathrm{HOIP}^{\mathrm{f} / \mathrm{fl}}$ mice infected with a lethal dose of WSN had significantly reduced mRNA expression of inflammatory cytokines, while total BALF cytokine concentrations were comparable. Thus, we believe another cell type is driving the inflammatory response to IAV infection in $\mathrm{SPC}^{\mathrm{Cre}} / \mathrm{HOIP}^{\mathrm{fl} / \mathrm{fl}}$ mice. It has been shown by several groups, in several models (TNF- $\alpha$, TRAIL, cisplatin), that loss of HOIP from a cell sensitizes it to induction of cell death (51, $61,67,68)$. This enhanced cell death in the absence of HOIP may account for the alternative activation of inflammation, as dying cells can release damage-associated molecular patterns (DAMPs) that can induce inflammation (69). LUBAC inhibits several proteins involved in the induction of both apoptosis and necroptosis, as well as stabilization of antiapoptotic proteins, and expression of NF- $\mathrm{kB}-$ dependent prosurvival genes. As such, loss of HOIP from the respiratory epithelium may have pleiotropic effects. Influenza virus can induce intrinsic cell death pathways to cause epithelial cell death, which would release DAMPs that activate resident immune cells. As the epithelial barrier is damaged, inflammatory infiltrates can more easily fill the airspace and contribute to immunopathology.

Use of a constitutively active mutant of IKK $\beta$ to bypass linear ubiquitin-dependent signaling and restore the NF- $\kappa B$ pathway in HOIP-deficient cells improved survival and reduced lung injury in $\mathrm{SPC}^{\mathrm{Cre}} / \mathrm{HOIP}{ }^{\mathrm{f} / \mathrm{ll}}$ mice during IAV infection, further supporting the crucial role of LUBAC-dependent signaling in regulating the host response to IAV infection. $\mathrm{SPC}^{\mathrm{Cre}} / \mathrm{HOIP}^{\mathrm{fl} / \mathrm{fl}}$ mice reconstituted with constitutively active IKK $\beta$ have restored NF- $\kappa B$ signaling, including the production of IL- 6 and IFN- $\beta$ (Supplemental Figure 8, B and C). Thus, upon IAV infection, IKK $\beta$-CA-expressing $\mathrm{SPC}^{\mathrm{Cre}} / \mathrm{HOIP}^{\mathrm{f} / \mathrm{fl}}$ AECs are able to initiate host response by activating NF- $\kappa \mathrm{B}$ downstream of LUBAC. In addition to the restored epithelial cell-driven production of cytokines and IFNs, rescue of the NF- $\kappa B$ pathway may induce the upregulation of prosurvival genes to reduce IAVinduced cell death pathways and contribute to the improved survival in AdIKK $\beta$-CA-treated SPC ${ }^{\mathrm{Cre}} / \mathrm{HOIP}^{\mathrm{fl} / \mathrm{fl}}$ mice. Collectively, restoration of the NF- $\kappa \mathrm{B}$ pathway in $\mathrm{SPC}^{\mathrm{Cre}} / \mathrm{HOIP}^{\mathrm{f} / \mathrm{l}}$ respiratory epithelial cells shifts the origin of the inflammatory response to IAV back to the epithelium. Taken together, our data underscore the critical importance of LUBAC-mediated NF- $\mathrm{KB}$ signaling in the alveolar epithelium orchestration of the host response to viral infections.

In conclusion, we show that during IAV infection, the amplitude of the inflammatory response is modulated by alveolar epithelial LUBAC activity, which serves as a molecular rheostat and 
regulates the host response. While several chemical inhibitors as well as peptides that bind HOIP have been used to inhibit LUBAC activity in cell culture $(70-73)$ and in vitro assays $(74,75)$ and support the targetablility of LUBAC, we have demonstrated that complete loss of HOIP is detrimental during IAV infection; however, some destabilization of LUBAC through loss of HOIL-1L is beneficial. Thus, novel compounds that target LUBAC stability to modulate LUBAC activity may be therapeutically beneficial for the treatment of hyperinflammatory response during IAV infection, where a modest degree of host response is necessary.

\section{Methods}

Mouse strains. Mice were bred in a barrier facility at Northwestern University. SPC ${ }^{\text {Cre }} /$ Rbck1(HOIL-1L) ${ }^{\mathrm{fl} / \mathrm{fl}}$ mice on a C57BL/ 6 background were generated as previously described (31). Rnf31(HOIP) ${ }^{\mathrm{f} / \mathrm{fl}}$ mice were generated on a C57BL/6 background by Ozgene Pty Ltd. Briefly, a conditional allele of HOIP was generated by flanking of exon 6 with loxP sites, generating a translational frameshift and rendering downstream exons nonfunctional. A neomycin cassette flanked by FRT sites was used for selection in embryonic stem cells. Subsequent breeding of WT/flox to WT/flp mice removed the neomycin cassette. Heterozygous crossing bred out $f l p$ and generated homozygous $\mathrm{HOIP}^{\mathrm{fl} / \mathrm{ll}}$ mice. $\mathrm{HOIP}^{\mathrm{fl} / \mathrm{fl}}$ mice were then bred to SPC ${ }^{\mathrm{Cre}}$ mice for the specific deletion of HOIP from AECs. The SPC ${ }^{\mathrm{Cre}} / \mathrm{HOIP}^{\mathrm{fl} / \mathrm{ll}}$ colony was maintained by crossing of $\mathrm{HOIP}^{\mathrm{f} / \mathrm{fl}}$ to $\mathrm{SPC}^{\mathrm{Cre}} / \mathrm{HOIP}^{\mathrm{f} / / \mathrm{fl}}$. Deletion was confirmed through genotyping (Transnetyx) and by immunoblot of AT2 lysates

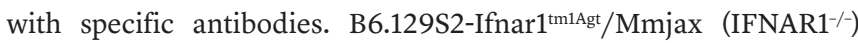
and B6.129S4-Ccr2 ${ }^{\text {tmlifc }} / \mathrm{J}$ (CCR2--) mice were purchased from The Jackson Laboratory and have been previously described $(76,77)$. Mice were provided with food and water ad libitum and maintained on a 14-hour light/10-hour dark cycle.

Histology. Lungs were fixed and processed as previously described (31). Bright-field images were obtained using TissueFAXS software (TissueGnostics). Quantification of severity of lung injury from H\&E images was based on peribronchial infiltration, bronchial exudate, and alveolar infiltration.

Reagents. The following pooled siRNAs were purchased from Santa Cruz Biotechnology (Scbt) and used at the indicated concentrations: siRBCK1(HOIL-1L) (sc-61446, 60 pmol), siIRF1 (sc-35706, 70 pmol), siSHARPIN (sc-77833, $120 \mathrm{pmol})$, siIFNAR1 (sc-35637, $70 \mathrm{pmol})$, and a scrambled nontargeting control (sc-37007) at corresponding concentrations. Additional reagents included siHOIP(RNF31) (Qiagen, 141713365; 120 pmol), Lipofectamine RNAiMAX (Thermo Fisher Scientific, 13778075), and recombinant human IFN- $\alpha$ (BioLegend, $592704 ; 100 \mathrm{U} / \mathrm{mL})$.

Cell culture. A549 (ATCC), MDCK (ATCC), and AT2 cells were isolated and cultured as previously described (31). Cell lines were routinely tested for mycoplasma contamination using the MycoAlert detection kit (Lonza).

Immunoprecipitation and immunoblot analysis. Immunoprecipitation was performed as previously described (78). Briefly, protein concentrations were incubated with A/G agarose beads (Scbt, sc-2003) and an anti-NEMO antibody (1 $\mu$; Scbt, sc-8330) rotating overnight. Beads were washed 3 times in 1\% wt/vol Triton X-100, 20 mM Tris$\mathrm{HCl}$ ( $\mathrm{pH}$ 7.5), $150 \mathrm{mM} \mathrm{NaCl}$, and eluted proteins analyzed by immunoblot. Immunoblotting was performed by resolving of protein lysates on SDS-PAGE gels (7.5\%-10\%), followed by transfer to nitrocellulose membranes (Bio-Rad) and further incubation of membranes with indicated antibodies overnight (Supplemental Table 1). HRP-tagged secondary antibodies (Bio-Rad, 1721011 and 1721019) were used in combination with Super-Signal ECL kit (Thermo Fisher Scientific) to develop blots using a LI-COR Odyssey Imager and companion software Image Studio version 5.2. Blots were quantified using densitometry (ImageJ 1.46r, NIH) (19).

IAV infection. Influenza A virus strain A/WSN/33(H1N1) was used for in vitro and in vivo experiments. A549 cells were seeded in 35-mm dishes at a density of $2 \times 10^{5}$ cells per well and infected at 1 MOI for 16 hours in DMEM. AT2 cells were seeded in a 12-well plate format at a density of $3 \times 10^{6}$ cells per well and infected at 1 MOI for 16 hours in DMEM. Conditioned medium was removed from infected cells and exposed to UV light for 15 minutes on ice for virus inactivation before addition to naive cells (79). As a control, UV-treated supernatant was boiled $\left(99^{\circ} \mathrm{C}\right)$ for 10 minutes to denature proteins.

For in vivo infections, anesthetized mice were inoculated intratracheally with WSN diluted in $50 \mu \mathrm{L}$ sterile PBS as previously described (80). A dose of $500 \mathrm{PFU}$ per mouse was used for lethal infections and $100 \mathrm{PFU}$ for the sublethal dose. For survival studies, upon developing a moribund condition (slowed respiration, hunched posture, lack of curiosity, little or no response to stimuli, and not moving when touched), mice were euthanized, and this was recorded as IAVinduced mortality. BALF was collected using $1 \mathrm{~mL}$ of PBS instilled into the lungs and aspirated 3 times $(81,82)$.

Determination of viral titer. Whole-lung homogenates serially diluted in DMEM containing 1\% BSA were added to MDCK cells, followed by a standard plaque assay protocol (83). Plaques were counted at each dilution to calculate viral titer.

Flow cytometry and cell sorting. Multicolor flow cytometry and cell sorting were performed with an LSR Fortessa or BD FACSAria cell sorter using DIVA software (BD Biosciences). Analysis was performed offline using FlowJo software (version 10.1). Myeloid and lymphocyte populations from whole lung were isolated and defined as previously described $(36,84,85)$. Briefly, perfused lungs were inflated with digestion buffer $(1 \mathrm{mg} / \mathrm{mL}$ of Collagenase D and $0.1 \mathrm{mg} / \mathrm{mL}$ DNase I, both from Roche) and coarsely minced before processing in C Tubes (Miltenyi Biotec) with a GentleMACS dissociator (Miltenyi Biotec), according to the manufacturer's instructions. Homogenate was passed through 40- $\mu$ m nylon mesh to obtain a single-cell suspension and subjected to red blood cell lysis (BD Pharm Lyse, BD Biosciences). Live cells were counted using a Countess cell counter (Invitrogen) by trypan blue exclusion.

Cells were stained with antibodies listed in Supplemental Tables 2 and 3 and gated as described elsewhere $(19,36)$ and outlined in Supplemental Figure 3, A and B. For lymphoid analysis, cells were first permeabilized with the FOXP3 staining kit (eBioscience, 005523) according to the manufacturer's instructions.

AT2 cells were isolated and sorted as previously described (12). Briefly, perfused lungs treated with dispase (Corning, 47743-724) and DNase (50 mg/mL; Sigma-Aldrich, D4513-1VL) were subjected to manual dissection. Single-cell suspensions were enriched for epithelial cells using anti-EpCAM magnetic microbeads (Miltenyi Biotec, 130105-958). Cells were stained with antibodies listed in Supplemental Table 4 and gated as outlined in Supplemental Figure 3C.

Native PAGE analysis. For analysis of native LUBAC formation, the Invitrogen Blue Native Gel system (Invitrogen, BN2007, BN2008) was 
used according to the manufacturer's instructions and as described elsewhere (86). Briefly, cells were lysed in provided sample buffer containing $1 \%$ digitonin and resolved on 3\%-12\% Bis-Tris gel (Invitrogen, BN1001). Proteins were transferred to a PVDF membrane and immunoblotted as described above.

Cytokine determination. ELISAs were carried out on cell culture supernatants or BALF according to the manufacturer's instructions using the following kits: IL-6(h) (Thermo Fisher Scientific, KHC0061C), IFN- $\beta$ (h) (PBL Assay Science, 41410), IL-6(m) (Thermo Fisher Scientific, KMC0062), MCP-1(m) (Thermo Fisher Scientific, EMMCP1), IFN- $\alpha(\mathrm{m})$ (PBL Assay Science, 42120), IFN- $\beta(\mathrm{m})$ (PBL Assay Science, 42400), IFN- $\gamma(\mathrm{m})$ (Thermo Fisher Scientific, BMS606), and IL-10(m) (Thermo Fisher Scientific, EM2IL10).

ChIP assay. Chromatin immunoprecipitation (ChIP) was performed using the Simple ChIP Enzymatic Chromatin IP kit (Cell Signaling, 9003) according to the manufacturer's instructions. Briefly, cells were cross-linked with formaldehyde and lysed with provided buffers. Cell lysates were subjected to sonication and nuclease treatment before immunoprecipitation with provided control IgG $(5 \mu \mathrm{g})$ or IRF1 (10 $\mu$ g; Scbt, sc-497) using provided magnetic beads. Bead eluates were subjected to proteinase digestion and quantitative PCR amplification of the HOIL-1L promoter (forward: 5'-TTAGCTTCAGTGTTCCCCCT-3'; reverse: 5'-CAGTGGGGAGACAATGAACAA-3') using SYBR Green (Bio-Rad, 1708880).

Quantitative reverse transcriptase PCR. Cellular mRNA was collected and purified using an RNeasy Mini Kit (Qiagen, 74104) before cDNA preparation with qScript cDNA synthesis kit (Quanta Bio, 95047). For quantitative reverse transcriptase PCR, IQ SYBR Green master mixes (Bio-Rad, 1708880) were used according to the manufacturer's instructions with the primers listed in Supplemental Table 5.

Statistics. Analyses of significance were performed using GraphPad Prism (version 7.02) software. A P value less than or equal to 0.05 was considered statistically significant. A standard 2-tailed unpaired Student's $t$ test was used for 2 groups. One-way ANOVA, followed by analysis-specific post-tests, was carried out when more than 2 variables were compared. Data are presented as means \pm SD overlaid with individual data points representing replicates. Statistical analysis of survival curves was performed with a 2-sided logrank (Mantel-Cox) test.
Study approval. All in vivo experiments were performed in compliance with the institutional and US NIH guidelines and were approved by the Northwestern University Animal Care and Use Committee.

\section{Author contributions}

PLB, LAD, and JIS designed research. PLB, LMN, NDM, and AVM performed research. KMR and KI contributed reagents/analytic tools. PLB, LAD, JGNG, AVM, GRSB, and JIS analyzed data. PLB, LAD, JIS, KMR, and GRSB discussed and edited the manuscript. PLB, LAD, and JIS wrote the manuscript.

\section{Acknowledgments}

This work was supported in part by grants sponsored by the National Heart, Lung, and Blood Institute (HL071643, HL076139, and HL132454). KI is supported by KAKENHI from the Japan Society for the Promotion of Science (grant 24112002). We thank Mark Ciesielski for isolation of AT2 cells and Lynn C. Welch for her technical assistance. This work was also supported by the Northwestern University Flow Cytometry Core Facility, supported by National Cancer Institute (NCI) Cancer Center Support Grant CA060553. The BD FACSAria SORP system was purchased through an NIH grant (1S10OD011996-01). Histology services were provided by the Northwestern University Mouse Histology and Phenotyping Laboratory supported by NCI P30-CA060553 awarded to the Robert H. Lurie Comprehensive Cancer Center. This work was supported by Northwestern University's Center for Advanced Microscopy and a Cancer Center Support Grant (NCI CA060553).

Address correspondence to: Jacob I. Sznajder or Laura A. Dada, Division of Pulmonary and Critical Care Medicine, Northwestern University Feinberg School of Medicine, 303 E. Superior Street, Simpson Querrey 5-300, Chicago, Illinois 60611, USA. Phone: 312.908.7737; Email: j-sznajder@northwestern.edu (JIS). Phone: 312.503.5397; Email: lauradada@northwestern.edu (LAD).

NDM's present address is: Universidad de Buenos Aires, CONICET, Instituto de Bioquímica y Medicina Molecular (IBIMOL), Departamento de Química Analítica y Fisicoquímica, Cátedra de Química General e Inorgánica Facultad de Farmacia y Bioquímica, Buenos Aires, Argentina.
1. Blank R, Napolitano LM. Epidemiology of ARDS and ALI. Crit Care Clin. 2011;27(3):439-458.

2. Jain S, et al. Hospitalized patients with 2009 $\mathrm{H} 1 \mathrm{~N} 1$ influenza in the United States, April-June 2009. N Engl J Med. 2009;361(20):1935-1944.

3. Napolitano LM, Park PK, Raghavendran K, Bartlett RH. Nonventilatory strategies for patients with life-threatening $2009 \mathrm{H} 1 \mathrm{~N} 1$ influenza and severe respiratory failure. Crit Care Med. 2010;38(4 suppl):e74-e90.

4. Rothberg MB, Haessler SD. Complications of seasonal and pandemic influenza. Crit Care Med. 2010;38(4 suppl):e91-e97.

5. Short KR, Kroeze EJBV, Fouchier RAM, Kuiken T. Pathogenesis of influenza-induced acute respiratory distress syndrome. Lancet Infect Dis. 2014;14(1):57-69.

6. Vadász I, Sznajder JI. Gas exchange disturbances regulate alveolar fluid clearance during acute lung injury. Front Immunol. 2017;8:757.

7. Herold S, Becker C, Ridge KM, Budinger GR. Influenza virus-induced lung injury: pathogenesis and implications for treatment. Eur Respir J. 2015;45(5):1463-1478.

8. Whitsett JA, Alenghat T. Respiratory epithelial cells orchestrate pulmonary innate immunity. Nat Immunol. 2015;16(1):27-35.

9. Sirén J, et al. Retinoic acid inducible gene-I and mda- 5 are involved in influenza A virus-induced expression of antiviral cytokines. Microbes Infect. 2006;8(8):2013-2020.

10. Kato $\mathrm{H}$, et al. Cell type-specific involvement of RIG-I in antiviral response. Immunity. 2005;23(1):19-28.

11. Davidson S, Crotta S, McCabe TM, Wack A. Pathogenic potential of interferon $\alpha \beta$ in acute influenza infection. Nat Commun. 2014;5:3864.

12. Peteranderl C, et al. Macrophage-epithelial paracrine crosstalk inhibits lung edema clearance during influenza infection. J Clin Invest. 2016;126(4):1566-1580.

13. Walsh KB, et al. Suppression of cytokine storm with a sphingosine analog provides protection against pathogenic influenza virus. Proc Natl Acad Sci U S A. 2011;108(29):12018-12023.

14. Lin KL, Suzuki Y, Nakano H, Ramsburg E, Gunn MD. CCR2+ monocyte-derived dendritic cells and exudate macrophages produce influenzainduced pulmonary immune pathology and mortality. JImmunol. 2008;180(4):2562-2572.

15. Arankalle VA, et al. Role of host immune response and viral load in the differential outcome of pandemic H1N1 (2009) influenza virus infection in Indian patients. PLoS One. 
2010;5(10):e13099.

16. de Jong MD, et al. Fatal outcome of human influenza A (H5N1) is associated with high viral load and hypercytokinemia. Nat Med. 2006;12(10):1203-1207.

17. Kobasa D, et al. Aberrant innate immune response in lethal infection of macaques with the 1918 influenza virus. Nature. 2007;445(7125):319-323.

18. Oldstone MB, Teijaro JR, Walsh KB, Rosen H. Dissecting influenza virus pathogenesis uncovers a novel chemical approach to combat the infection. Virology. 2013;435(1):92-101.

19. Brazee PL, et al. FXYD5 is an essential mediator of the inflammatory response during lung injury. Front Immunol. 2017;8:623.

20. Tokunaga F, Iwai K. LUBAC, a novel ubiquitin ligase for linear ubiquitination, is crucial for inflammation and immune responses. Microbes Infect. 2012;14(7-8):563-572.

21. Tokunaga $F$, et al. Involvement of linear polyubiquitylation of NEMO in NF-KB activation. Nat Cell Biol. 2009;11(2):123-132.

22. Smit JJ, Monteferrario D, Noordermeer SM, van Dijk WJ, van der Reijden BA, Sixma TK. The E3 ligase HOIP specifies linear ubiquitin chain assembly through its RING-IBR-RING domain and the unique LDD extension. EMBO J. 2012;31(19):3833-3844.

23. Brazee P, Dada LA, Sznajder JI. Role of linear ubiquitination in health and disease. Am J Respir Cell Mol Biol. 2016;54(6):761-768.

24. Haas TL, et al. Recruitment of the linear ubiquitin chain assembly complex stabilizes the TNF-R1 signaling complex and is required for TNF-mediated gene induction. Mol Cell. 2009;36(5):831-844.

25. Kirisako T, et al. A ubiquitin ligase complex assembles linear polyubiquitin chains. $E M B O J$. 2006;25(20):4877-4887.

26. Ikeda $F$, et al. SHARPIN forms a linear ubiquitin ligase complex regulating NF- $\mathrm{KB}$ activity and apoptosis. Nature. 2011;471(7340):637-641.

27. Stieglitz B, Morris-Davies AC, Koliopoulos MG, Christodoulou E, Rittinger K. LUBAC synthesizes linear ubiquitin chains via a thioester intermediate. EMBO Rep. 2012;13(9):840-846.

28. Rahighi S, et al. Specific recognition of linear ubiquitin chains by NEMO is important for NF-КB activation. Cell. 2009;136(6):1098-1109.

29. Tarantino N, et al. TNF and IL-1 exhibit distinct ubiquitin requirements for inducing NEMOIKK supramolecular structures. JCell Biol. 2014;204(2):231-245.

30. Deckers J, Branco Madeira F, Hammad H. Innate immune cells in asthma. Trends Immunol. 2013;34(11):540-547.

31. Magnani ND, et al. HIF and HOIL-1L-mediated PKC $\zeta$ degradation stabilizes plasma membrane $\mathrm{Na}$,K-ATPase to protect against hypoxiainduced lung injury. Proc Natl Acad Sci U S A. 2017;114(47):E10178-E10186.

32. Tokunaga $\mathrm{F}$, et al. SHARPIN is a component of the NF-KB-activating linear ubiquitin chain assembly complex. Nature. 2011;471(7340):633-636.

33. Fujita $\mathrm{H}$, et al. Cooperative domain formation by homologous motifs in HOIL-1L and SHARPIN plays a crucial role in LUBAC stabilization. Cell Rep. 2018;23(4):1192-1204.
34. Peltzer N, et al. LUBAC is essential for embryogenesis by preventing cell death and enabling haematopoiesis. Nature. 2018;557(7703):112-117.

35. Elton L, Carpentier I, Verhelst K, Staal J, Beyaert R. The multifaceted role of the E3 ubiquitin ligase HOIL-1: beyond linear ubiquitination. Immunol Rev. 2015;266(1):208-221.

36. Misharin AV, Morales-Nebreda L, Mutlu GM, Budinger GR, Perlman H. Flow cytometric analysis of macrophages and dendritic cell subsets in the mouse lung. Am J Respir Cell Mol Biol. 2013;49(4):503-510.

37. Braciale TJ, Sun J, Kim TS. Regulating the adaptive immune response to respiratory virus infection. Nat Rev Immunol. 2012;12(4):295-305.

38. Pulendran B, Maddur MS. Innate immune sensing and response to influenza. Curr Top Microbiol Immunol. 2015;386:23-71.

39. Queisser MA, et al. HOIL-1L functions as the PKC $\zeta$ ubiquitin ligase to promote lung tumor growth. Am J Respir Crit Care Med. 2014;190(6):688-698.

40. Hui KP, et al. H5N1 influenza virus-induced mediators upregulate RIG-I in uninfected cells by paracrine effects contributing to amplified cytokine cascades. J Infect Dis. 2011;204(12):1866-1878.

41. Seng LG, Daly J, Chang KC, Kuchipudi SV. High basal expression of interferon-stimulated genes in human bronchial epithelial (BEAS-2B) cells contributes to influenza A virus resistance. PLoS One. 2014;9(10):e109023.

42. Portela A, Digard P. The influenza virus nucleoprotein: a multifunctional RNA-binding protein pivotal to virus replication. J Gen Virol. 2002;83(pt 4):723-734.

43. Tamiya $\mathrm{H}$, et al. IFN- $\gamma$ or IFN- $\alpha$ ameliorates chronic proliferative dermatitis by inducing expression of linear ubiquitin chain assembly complex. J Immunol. 2014;192(8):3793-3804.

44. Andrilenas KK, Ramlall V, Kurland J, Leung B, Harbaugh AG, Siggers T. DNA-binding landscape of IRF3, IRF5 and IRF7 dimers: implications for dimer-specific gene regulation. Nucleic Acids Res. 2018;46(5):2509-2520.

45. Ozato K, Tailor P, Kubota T. The interferon regulatory factor family in host defense: mechanism of action. J Biol Chem. 2007;282(28):20065-20069.

46. Messeguer X, Escudero R, Farré D, Núñez $\mathrm{O}$, Martínez J, Albà MM. PROMO: detection of known transcription regulatory elements using species-tailored searches. Bioinformatics. 2002;18(2):333-334

47. Bime C, et al. Genome-wide association study in African Americans with acute respiratory distress syndrome identifies the selectin P ligand gene as a risk factor. Am J Respir Crit Care Med. 2018;197(11):1421-1432.

48. Otero JE, Dai S, Alhawagri MA, Darwech I, Abu-Amer Y. IKKbeta activation is sufficient for RANK-independent osteoclast differentiation and osteolysis. JBone Miner Res. 2010;25(6):1282-1294.

49. Lawrence $\mathrm{T}$. The nuclear factor NF- $\mathrm{kB}$ pathway in inflammation. Cold Spring Harb Perspect Biol. 2009;1(6):a001651.

50. Krammer F, Palese P. Advances in the development of influenza virus vaccines. Nat Rev Drug
Discov. 2015;14(3):167-182.

51. Lafont $\mathrm{E}$, et al. The linear ubiquitin chain assembly complex regulates TRAIL-induced gene activation and cell death. ЕMBO J. 2017;36(9):1147-1166.

52. Yarilina A, Park-Min KH, Antoniv T, Hu X, Ivashkiv LB. TNF activates an IRF1-dependent autocrine loop leading to sustained expression of chemokines and STAT1-dependent type I interferon-response genes. Nat Immunol. 2008;9(4):378-387.

53. Ivashkiv LB, Donlin LT. Regulation of type I interferon responses. Nat Rev Immunol. 2014;14(1):36-49.

54. Balachandran S, Beg AA. Defining emerging roles for $\mathrm{NF}-\mathrm{KB}$ in antivirus responses: revisiting the interferon- $\beta$ enhanceosome paradigm. PLOS Pathog. 2011;7(10):e1002165.

55. Szretter KJ, et al. Early control of H5N1 influenza virus replication by the type I interferon response in mice. JVirol. 2009;83(11):5825-5834.

56. Herold S, et al. Lung epithelial apoptosis in influenza virus pneumonia: the role of macrophage-expressed TNF-related apoptosis-inducing ligand. J Exp Med. 2008;205(13):3065-3077.

57. Shahangian A, et al. Type I IFNs mediate development of postinfluenza bacterial pneumonia in mice. JClin Invest. 2009;119(7):1910-1920.

58. Inn KS, et al. Linear ubiquitin assembly complex negatively regulates RIG-I- and TRIM25mediated type I interferon induction. Mol Cell. 2011;41(3):354-365.

59. Belgnaoui SM, et al. Linear ubiquitination of NEMO negatively regulates the interferon antiviral response through disruption of the MAVS-TRAF3 complex. Cell Host Microbe. 2012;12(2):211-222.

60. Boisson B, et al. Immunodeficiency, autoinflammation and amylopectinosis in humans with inherited HOIL-1 and LUBAC deficiency. Nat Immunol. 2012;13(12):1178-1186.

61. Zinngrebe J, et al. LUBAC deficiency perturbs TLR3 signaling to cause immunodeficiency and autoinflammation.JExp Med 2016;213(12):2671-2689.

62. Zhou G, Juang SW, Kane KP. NK cells exacerbate the pathology of influenza virus infection in mice. Eur JImmunol. 2013;43(4):929-938.

63. Carlin LE, Hemann EA, Zacharias ZR, Heusel JW, Legge KL. Natural killer cell recruitment to the lung during influenza A virus infection is dependent on CXCR3, CCR5, and virus exposure dose. Front Immunol. 2018;9:781.

64. Duan S, Thomas PG. Balancing immune protection and immune pathology by CD8(+) T-cell responses to influenza infection. Front Immunol. 2016;7:25.

65. Wells MA, Albrecht P, Ennis FA. Recovery from a viral respiratory infection. I. Influenza pneumonia in normal and T-deficient mice. J Immunol. 1981;126(3):1036-1041.

66. Arpaia N, et al. A distinct function of regulatory $\mathrm{T}$ cells in tissue protection. Cell. 2015;162(5):1078-1089.

67. MacKay C, et al. E3 ubiquitin ligase HOIP attenuates apoptotic cell death induced by cisplatin. Cancer Res. 2014;74(8):2246-2257.

68. Tang $\mathrm{Y}$, et al. Linear ubiquitination of cFLIP induced by LUBAC contributes to 
TNF $\alpha$-induced apoptosis. J Biol Chem. 2018;293(52):20062-20072.

69. Fujikura D, Miyazaki T. Programmed cell death in the pathogenesis of influenza. Int J Mol Sci. 2018;19(7):E2065.

70. Aguilar-Alonso F, Whiting AL, Kim YJ, Bernal F. Biophysical and biological evaluation of optimized stapled peptide inhibitors of the linear ubiquitin chain assembly complex (LUBAC). Bioorg Med Chem. 2018;26(6):1179-1188.

71. Katsuya K, et al. Small-molecule inhibitors of linear ubiquitin chain assembly complex (LUBAC), HOIPINs, suppress NF-kB signaling. Biochem Biophys Res Commun. 2019;509(3):700-706.

72. Strickson $S$, et al. The anti-inflammatory drug BAY 11-7082 suppresses the MyD88-dependent signalling network by targeting the ubiquitin system. Biochem J. 2013;451(3):427-437.

73. Yang Y, et al. Essential role of the linear ubiquitin chain assembly complex in lymphoma revealed by rare germline polymorphisms. Cancer Discov. 2014;4(4):480-493.

74. Johansson H, et al. Fragment-based covalent ligand screening enables rapid discovery of inhibitors for the RBR E3 ubiquitin ligase HOIP. JAm Chem Soc. 2019;141(6):2703-2712.

75. Sakamoto H, et al. Gliotoxin suppresses NF- $\mathrm{\kappa B}$ activation by selectively inhibiting linear ubiquitin chain assembly complex (LUBAC). ACS Chem Biol. 2015;10(3):675-681.

76. Müller U, et al. Functional role of type I and type II interferons in antiviral defense. Science. 1994;264(5167):1918-1921.

77. Boring L, et al. Impaired monocyte migration and reduced type 1 (Th1) cytokine responses in C-C chemokine receptor 2 knockout mice. J Clin Invest. 1997;100(10):2552-2561.

78. Sasaki Y, Fujita H, Nakai M, Iwai K. Immunoblot analysis of linear polyubiquitination of NEMO. Methods Mol Biol. 2015;1280:297-309.

79. Guo X, et al. Respiratory syncytial virus infection upregulates NLRC5 and major histocompatibility complex class I expression through RIG-I induction in airway epithelial cells. J Virol. 2015;89(15):7636-7645.

80. Morales-Nebreda LI, et al. Lung-specific loss of $\alpha 3$ laminin worsens bleomycin-induced pulmonary fibrosis. Am J Respir Cell Mol Biol. 2015;52(4):503-512.

81. Lubarski-Gotliv I, Asher C, Dada LA, Garty H. FXYD5 protein has a pro-inflammatory role in epithelial cells. J Biol Chem. 2016;291(21):11072-11082.

82. Urich D, et al. Lung-specific loss of the laminin $\alpha 3$ subunit confers resistance to mechanical injury. JCell Sci. 2011;124(pt 17):2927-2937.

83. Baer A, Kehn-Hall K. Viral concentration determination through plaque assays: using traditional and novel overlay systems. J Vis Exp. 2014;(93):e52065.

84. Dillon SR, et al. Interleukin 31, a cytokine produced by activated $\mathrm{T}$ cells, induces dermatitis in mice. Nat Immunol. 2004;5(7):752-760.

85. D'Alessio FR, et al. CD $4^{+} \mathrm{CD} 25^{+} \mathrm{Foxp}^{+}{ }^{+}$Tregs resolve experimental lusng injury in mice and are present in humans with acute lung injury. JClin Invest. 2009;119(10):2898-2913.

86. Wittig I, Braun HP, Schägger H. Blue native PAGE. Nat Protoc. 2006;1(1):418-428. 